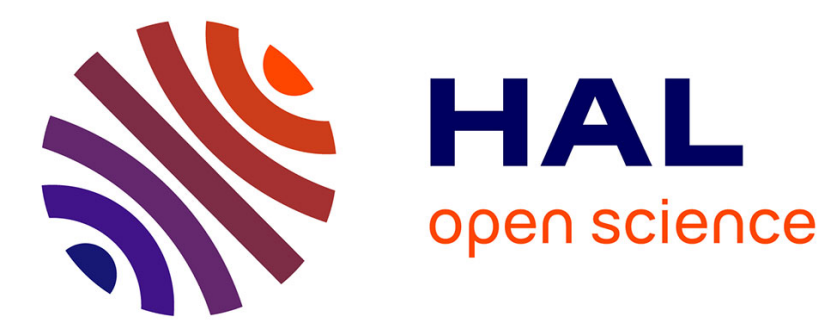

\title{
The formation of tabular compaction-band arrays: Theoretical and numerical analysis
}

\author{
Alexandre I. Chemenda
}

\section{To cite this version:}

Alexandre I. Chemenda. The formation of tabular compaction-band arrays: Theoretical and numerical analysis. Journal of the Mechanics and Physics of Solids, 2009, 57 (5), pp.851-868. 10.1016/j.jmps.2009.01.007 . hal-00407974

\section{HAL Id: hal-00407974 https://hal.science/hal-00407974}

Submitted on 25 Jul 2019

HAL is a multi-disciplinary open access archive for the deposit and dissemination of scientific research documents, whether they are published or not. The documents may come from teaching and research institutions in France or abroad, or from public or private research centers.
L'archive ouverte pluridisciplinaire HAL, est destinée au dépôt et à la diffusion de documents scientifiques de niveau recherche, publiés ou non, émanant des établissements d'enseignement et de recherche français ou étrangers, des laboratoires publics ou privés. 


\title{
The formation of tabular compaction-band arrays: Theoretical and numerical analysis
}

\author{
A.I. Chemenda * \\ Géosciences Azur, Université de Nice-Sophia Antipolis, CNRS, 250 Rue Albert Einstein, 06560 Valbonne, France
}

\begin{abstract}
The bifurcation analysis of compaction banding is extended to the formation of a tabular discrete compaction-band array. This analysis, taken together with the results of finitedifference simulations, shows that the bifurcation results in the formation of intermittent loading (elastic-plastic) and unloading (elastic) bands. The obtained analytical solution relates the spacing parameter $\chi$ (the ratio between the band thickness to the band-to-band distance) to all constitutive and stress-state parameters. Both this solution and numerical models reveal strong dependence of $\chi$ on the hardening modulus $h: \chi$ increases with $h$ reduction. The band thickness in the numerical models is mesh dependent, but in terms of mesh-zone-size varies only from $\sim 2$ to 4 depending on the constitutive parameters and independently on the mesh resolution. The thickness of the "elementary" compaction bands in real granular materials is equal to a few grain sizes. It follows that one grid zone in the numerical models corresponds approximately to one grain in the real material. The numerical models reproduce both discrete and continuous propagating compaction banding observed in the rock samples. These phenomena were shown to be dependent on the evolution of $h$ and the dilatancy factor with deformation.
\end{abstract}

\section{Introduction}

The bifurcation theory (Hill, 1962; Mandel, 1964, 1966; Rudnicki and Rice, 1975; Rice, 1976) was originally applied to the formation of shear localization bands (slip surfaces, Lüders lines). Perrin and Leblond (1993) have shown that bifurcation from homogeneous deformation can also result in formation of planar localization bands without shear deformation within them. This issue was then analyzed in more detail in Issen and Rudnicki (2000) and Bésuelle (2001).

The non-shear bands can be either parallel (compaction bands) or orthogonal (dilation bands) to the least compressive stress $\sigma_{1}$, with all band types being parallel to the intermediate stress direction ( $\sigma_{i}$ are the principal stresses: $\sigma_{3} \leqslant \sigma_{2} \leqslant \sigma_{1}$; the compressive stress is negative, $i=1,2,3)$. Most studies deal with the compaction bands since they have been clearly observed in nature (e.g., Mollema and Antonellini, 1996; Sternlof et al., 2004) and were reproduced in laboratory in geomaterial samples (e.g., Olsson and Holcomb, 2000; Klein et al., 2001; Wong et al., 2001; Baud et al., 2004, 2006; Fortin et al., 2006) as well as in other materials such as metal foams (Bastawros et al., 2000), polycarbonate honeycomb (Papka and Kyriakides, 1999), etc.

As in the case of shear bands, the analysis of the compaction banding in the previous works was limited to the continuous bifurcation and so did not deal with the formation of discrete bands (band sets). On the other hand, it is such

\footnotetext{
* Tel.: +33492942661; fax: +33492942610.

E-mail address: chem@geoazur.unice.fr
} 


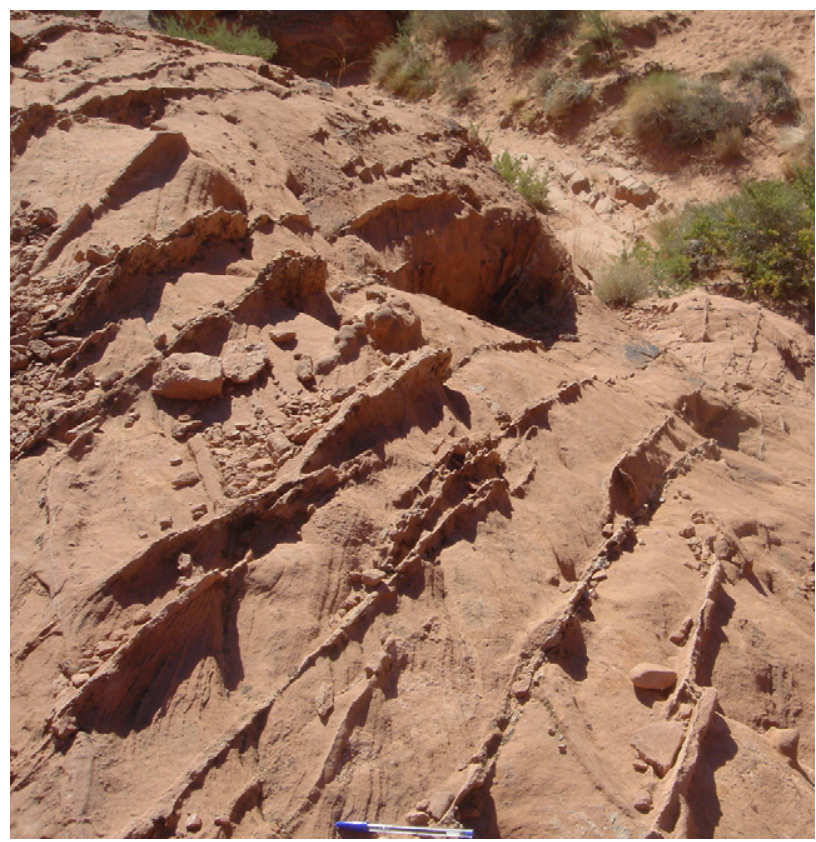

Fig. 1. Compaction bands in Aztec Sandstone, the Valley of Fire State Park, Nevada (for geological details of the site see e.g. (Sternlof et al., 2004). Pen at the bottom of the photo for scale.

sets (tabular arrays) that are typically observed in nature (Fig. 1). These arrays are characterized by a certain dominant (for each case) spacing $\lambda$. This parameter $(\lambda)$ is very important both for reservoir applications (e.g., hydraulic compartitioning and permeability) and interpretation of the experimental data, and is certainly defined by the material properties and the stress state.

In the multi-shear-band analysis corresponding to the formation of a localization band network accompanied by the elastic unloading of the material outside the bands, Chemenda (2007) found that $\lambda$ is related to the band thickness $d$. Both parameters enter the bifurcation condition as a ratio $\chi=d / \lambda$ and cannot be separated since the classical phenomenological plasticity theory used does not have a spatial scale. More complete theory based on the micro-physical analysis should contain such a scale (the grain size, for example). The integration of the material microstructure is attempted in more sophisticated (gradient, non-local, etc.) constitutive formulations (see Vardoulakis and Sulem (1995) and Bésuelle and Rudnicki (2004) for a review), but the parameters of these models are very poorly constrained by observations, which renders their practical use problematic.

There remains a possibility to estimate $\lambda$ from the above-mentioned multi-band analysis by assigning a certain realistic value to $d$ which varies little, whether in the field examples or in the laboratory samples, and typically equals a few to several grain sizes. The multi-band analysis has been performed for an arbitrary orientation in the stress space of the network of conjugated shear bands which can be pure shear, shear-dilatation or shear-compaction (Chemenda, 2007). The obtained solution relating $\chi$ to the constitutive and stress-state parameters at bifurcation is, however, not valid for the band sets parallel and orthogonal to $\sigma_{3}$ : the transition from the conjugated, oblique to $\sigma_{3}$ band network to the orthogonal/ parallel to $\sigma_{3}$ band sets is not as straightforward as in the case of continuous bifurcation analysis where the transition is continuous (Bésuelle, 2001).

The present paper deals with the case when the band set is orthogonal to $\sigma_{3}$ (all band types are also parallel to $\sigma_{2}$ ) which corresponds to the compaction banding. The analysis is much simpler than for shear banding since the bands lie in the principal plan $\left(\sigma_{1}, \sigma_{2}\right)$ and there is neither rigid body spins nor stress axe rotation during bifurcation. Two cases are considered: one when the bifurcation occurs in the regime of continuing loading, with the whole body remaining in the elastic-plastic state (continuous bifurcation), and the other, when the inter-band zones undergo the elastic unloading during bifurcation (discontinuous bifurcation). For the continuous bifurcation we obtained the same critical condition as Perrin and Leblond (1993) and in Issen and Rudnicki (2000) and Bésuelle (2001). For the discontinuous bifurcation, the critical condition contains a spacing parameter $\chi=d / \lambda$ which is a function of stress state and all constitutive parameters including the hardening modulus $h$. As for shear banding, $\chi$ increases ( $\lambda$ reduces) with $h$ reduction. The compaction banding is possible when the constitutive parameter values are in a certain range, which is much wider than for the continuous bifurcation. It follows that compaction banding can occur at much larger (smaller negative or even positive) dilatancy factor values than those predicted by the continuous bifurcation analysis. These values correspond well to those for geomaterials (Wong et al., 2001; Baud et al., 2006). The theoretical results are confirmed by the finite-difference numerical models which also reveal a predicted strong dependence of $\lambda$ on the constitutive and stress-state parameters. 
The numerical models reproduce both discrete and continuous propagating banding observed in the rock samples (Klein et al., 2001; Wong et al., 2001; Olsson, 2001; Baud et al., 2004).

\section{Velocity field at the onset of the formation of the compaction bands}

Consider conditions for bifurcation of the homogeneous deformation field into a regular deformation pattern in Fig. 2 a. This pattern is formed under initially uniform stressing by a set of compaction bands parallel to $x_{1}$ (axes $x_{i}$ are oriented in the principal directions). One can outline an elementary deformation element (or cell) of which the whole pattern can be composed (Fig. 2b). Limit the analysis to this cell. Consider the perturbation $v_{i}$ of the velocity field caused by the deformation bifurcation ( $v_{i}$ is the difference between the velocity field at the onset of bifurcation and the field just before the bifurcation). Both $v_{i}$ and their spatial derivatives $v_{i, j}(j=1,2,3)$ are non-zero only in the direction orthogonal to the bands. The velocities $v_{i}$ at the cell boundaries are indicated in Fig. 2b. Assume that the deformation field is uniform both within the localization band and outside it. The definition of the velocity gradient field within the cell is then straightforward from Fig. 2b:

$$
\begin{aligned}
& v_{3,3}^{(1)}=v_{b} / d, \\
& v_{3,3}^{(2)}=-v_{b} / \lambda, \\
& v_{k, l}^{(r)}=0, \quad \text { if } k \neq l \neq 3,
\end{aligned}
$$

where $r=1$, 2, with $r=1$ standing for the localization band, and $r=2$ for the inter-band zone; $k, l=1,2,3$.

\section{Bifurcation condition}

The following is based on Hill $(1962)$, Mandel $(1964,1966)$ and Rudnicki and Rice (1975). Since both the properties and the deformation field are uniform within the localization band and outside it, the stress field is uniform as well. Therefore, the equilibrium equations are met in both zones and hence the stress can change only across the band boundary. Writing the equations of continuing equilibrium in terms of first Piola-Kirchhoff stress $\pi_{i j}, \pi_{i j, i}=0$ (Hill, 1962) and considering that at the onset of bifurcation the stress can change only at the band boundary in a direction perpendicular to it obtain

$$
\dot{\pi}_{33,3}=0
$$

where

$$
\dot{\pi}_{i j}=\dot{\sigma}_{i j}+\sigma_{i j} v_{k, k}-\sigma_{k j} v_{i, k},
$$

and $\dot{\sigma}_{i j}$ is the material time derivatives of the Cauchy stress $\sigma_{i j}$. From (2) follows the condition of the continuity of the traction rate at the band boundary:

$$
\dot{\pi}_{33}^{(1)}-\dot{\pi}_{33}^{(2)}=0,
$$

a

b

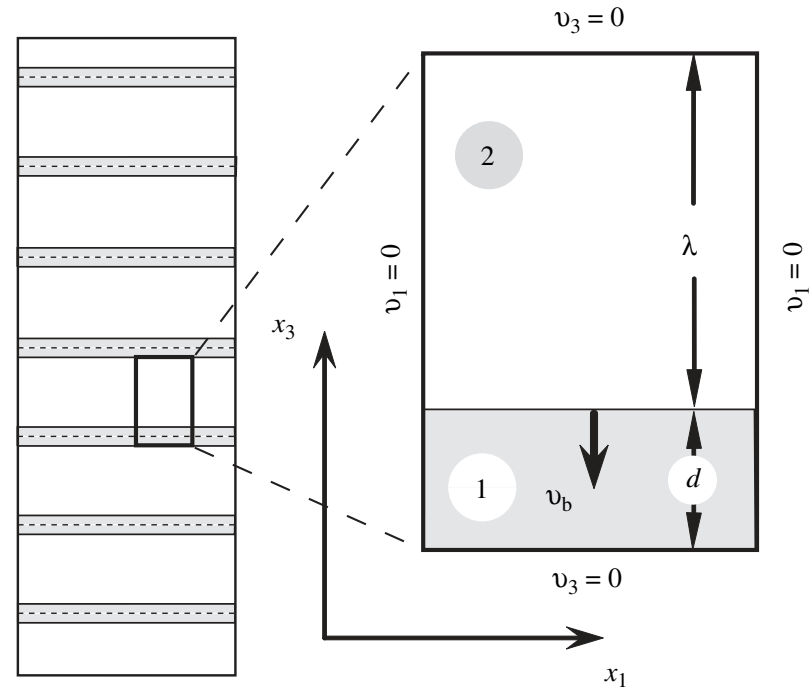

Fig. 2. Regular set of compaction bands (a) and an elementary cell of the deformation pattern (b). $d$ is the localization band thickness; $\lambda$ is the inter-band distance; $v_{\mathrm{b}}$ is the velocity vector at the compaction band boundary during bifurcation relative to $x_{1}$-parallel cell boundaries ( $v_{\mathrm{b}}$ is orthogonal to the band); $x_{i}$ are the principal axes. 1 and 2 denote localization band and inter-band zones, respectively. 
where $\dot{\pi}_{33}^{(r)}$ are stresses at the internal $(r=1)$ and external $(r=2)$ sides of the localization band, respectively. The strain and deformation rates are related by a linear, homogeneous tensor relation:

$$
{\stackrel{\nabla}{\sigma_{i j}}}^{(r)}=L_{i j k l}^{(r)} D_{k l}^{(r)},
$$

where $D_{i j}=(1 / 2)\left(v_{i, j}+v_{j, i}\right) ; \stackrel{\nabla}{\sigma}_{i j}$ is the invariant to rigid body spins Jaumann derivative of the Cauchy stress:

$$
\stackrel{\nabla}{\sigma}_{i j}=\dot{\sigma}_{i j}-W_{i k} \sigma_{k j}+\sigma_{i k} W_{k j} \text {, }
$$

$W_{i j}=(1 / 2)\left(v_{i, j}-v_{j, i}\right)$ and $L_{i j k l}$ is the incremental elastic-plastic stiffness tensor.

Substituting the velocity gradient from (1) into (3) and (6) obtain $\dot{\pi}_{i j}=\sigma_{i j}=\dot{\sigma}_{i j}$ which simplifies (4) and (5) to their usual form while neither neglecting the rotational effects nor assuming that the acting stresses are small compared to the stiffness moduli (this is true only when the localization bands are parallel to a principal plane when the rotation of the principal axes does not occur during the bifurcation). Instead of (4) and (5) we thus have, respectively

$$
\dot{\sigma}_{33}^{(1)}-\dot{\sigma}_{33}^{(2)}=0
$$

and

$$
\dot{\sigma}_{i j}^{(r)}=\frac{1}{2} L_{i j k l}^{(r)}\left(v_{k, l}^{(r)}+v_{l, k}^{(r)}\right) .
$$

Substituting (1) and (8) into (7) yields the bifurcation condition:

$$
L_{3333}^{(1)}+\chi L_{3333}^{(2)}=0 \text {, }
$$

where $\chi=d / \lambda$ is the spacing parameter. This equation can be also obtained from the general formulas derived by Garagash (1981) based on the virtual work principle for a set of parallel bands (he considered shear bands arbitrarily oriented in $\left(x_{1}, x_{3}\right)$ plan).

To specify the stiffness tensors $L_{i j k l}^{(r)}$, assume the isotropic-hardening model with Drucker-Prager both yield $f\left(\sigma_{i j}\right)$ and plastic potential $\Phi\left(\sigma_{i j}\right)$ functions:

$$
\begin{aligned}
& f=\bar{\tau}+\alpha \sigma-k, \\
& \Phi=\bar{\tau}+\beta \sigma,
\end{aligned}
$$

where $\alpha$ is the internal friction coefficient; $k$ is the cohesion; $\beta$ is the dilatancy factor; $\bar{\tau}=\left((1 / 2) s_{i j} s_{i j}\right)^{1 / 2}$ is the Mises equivalent shear stress; $s_{i j}=\sigma_{i j}-\delta_{i j} \sigma$ is the stress deviator, $\delta_{i j}$ is the Kronecker delta, and $\sigma=(1 / 3) \sigma_{i i}$ is the mean stress. This model, along with Hooke's equations, results in the following expressions for $L_{i j k l}$ (Rudnicki and Rice, 1975):

$$
L_{i j k l}=G\left\{\left[\left(\delta_{i k} \delta_{j l}+\delta_{i l} \delta_{k j}\right)+\left(\frac{K}{G}-\frac{2}{3}\right) \delta_{k l} \delta_{i j}\right]-\frac{G}{(H+G)+\alpha \beta K}\left(N_{i j}+\frac{K}{G} \beta \delta_{i j}\right)\left(N_{k l}+\frac{K}{G} \alpha \delta_{k l}\right)\right\},
$$

where $K$ and $G$ are the bulk and shear elastic moduli, respectively; $N_{i j}=s_{i j} / \bar{\tau} ; H=d k / d \bar{\gamma}^{p}-\sigma\left(d \alpha / d \bar{\gamma}^{p}\right)$ is the plastic hardening modulus; $d \bar{\gamma}^{p}=\left(2 d e_{i j}^{p} d e_{i j}^{p}\right)^{1 / 2}, d e_{i j}{ }^{p}=d \varepsilon_{i j}{ }^{p}-(1 / 3) d \varepsilon^{p}, d \varepsilon_{i j}{ }^{p}$ is the increment of inelastic strain, and $d \varepsilon^{p}=d \varepsilon_{i i}{ }^{p}$. The dilatancy factor is defined as $\beta=d \varepsilon^{p} / d \bar{\gamma}^{p}$ (Nikolaevskiy, 1967).

\section{Continuous bifurcation}

In this case the material within and outside the localization bands is in elastic-plastic state and $L_{i j k l}{ }^{(1)}=L_{i j k l}{ }^{(2)}=L_{i j k l}$ where $L_{i j k l}$ is given by (11). The bifurcation condition (9) is transformed to

$$
L_{3333}(1+\chi)=0
$$

which is satisfied when $L_{3333}=0$. Solving this equation for the hardening modulus yields

$$
h_{c r}^{c}=\frac{H_{c r}^{c}}{G}=\frac{(1+v)(\beta-\alpha)^{2}}{9(1-v)}-\frac{1+v}{1-v}\left(\frac{1}{2} N_{3}-\frac{\alpha+\beta}{3}\right)^{2}-\left(1-\frac{3}{4} N_{3}^{2}\right)
$$

where $N_{3}=\left(s_{3} / \bar{\tau}\right)=-(1 / 2)\left(N+(1 / 2) \sqrt{4-3 N^{2}}\right), N=s_{2} / \bar{\tau}, s_{i}$ is the principal deviatoric stress, superscript " $c$ " stands for compaction.

When the reducing normalized hardening modulus $h$ reaches $h_{c r}^{c}$ during inelastic deformation, the uniform deformation field should bifurcate resulting in formation of the compaction bands. This can occur only if $h_{c r}^{c}$ is not less than the critical hardening modulus $h_{c r}^{\text {sh }}$ at which the loss of stability takes place in the form of shear banding

$$
h_{c r}^{c} \geqslant h_{c r}^{s h},
$$

otherwise the deformation localization will occur via shear banding. For the continuous bifurcation, $h_{c r}^{\text {sh }}$ obtained by Rudnicki and Rice (1975) for a single shear band was shown to be the same as for the network of conjugated shear bands 
(Chemenda, 2007) and equal to

$$
h_{c r}^{s h}=\frac{H_{c r}^{s h}}{G}=\frac{1+v}{9(1-v)}(\beta-\alpha)^{2}-\frac{1+v}{2}\left(N+\frac{\alpha+\beta}{3}\right)^{2} .
$$

Substituting (13) and (15) into (14) yields

$$
N_{3} \geqslant \frac{(1+v)(\alpha+\beta)}{3}-(1-v) N
$$

or

$$
\beta \leqslant-\frac{\alpha(1+v)+3(v-1) N-3 N_{3}}{(1+v)}
$$

or

$$
\alpha \leqslant-\frac{\beta(1+v)+3(v-1) N-3 N_{3}}{(1+v)} .
$$

Expressions (13) and (16) are equivalent to those obtained in (Perrin and Leblond, 1993; Issen and Rudnicki, 2000; Bésuelle, 2001) which is not surprising since they follow from (12) equivalent in turn to the condition of vanishing the determinant of the acoustic tensor det $\mid n_{i} L_{i j k l} n_{l}=0$ for a compaction band, with the unit normal $n_{i}$ in the principal axes being: $n_{1}=n_{2}=0 ; n_{3}=1$.

Although the band spacing parameter $\chi$ is present in Eq. (12), it does not enter the final expressions (13) and (16) and hence is not defined, similar to the shear-banding problem (Chemenda, 2007).

The deformation accelerates within the localization (loading) bands and undergoes deceleration (unloading) outside them. Therefore at the next stage of deformation (infinitesimal deformation past the continuous bifurcation point), the constitutive response outside the bands should become elastic (Rice and Rudnicki, 1980) which corresponds to the discontinuous or elastic-plastic bifurcation (Rice and Rudnicki, 1980; Ottosen and Runesson, 1991).

\section{Discontinuous bifurcation}

In this case $L_{i j k l}^{(1)}=L_{i j k l}$ from (11) and

$$
L_{i j k l}^{(2)}=G\left[\left(\delta_{i k} \delta_{j l}+\delta_{i l} \delta_{k j}\right)+\left(\frac{K}{G}-\frac{2}{3}\right) \delta_{k l} \delta_{i j}\right] .
$$

Substituting these into (9) and solving it for $h$ and $\chi$ yields

$$
\begin{aligned}
& h=\frac{H}{G}=h_{c r}^{c}+\chi \frac{C_{\alpha \beta}}{18(2 v-1)(1-v)(\chi+1)}, \\
& \chi=\frac{18\left(h-h_{c r}^{c}\right)(2 v-1)(1-v)}{18\left(h-h_{c r}^{c}\right)(2 v-1)(v-1)+C_{\alpha \beta}},
\end{aligned}
$$

where $C_{\alpha \beta}=\left[2 \beta(1+v)-3 N_{3}(2 v-1)\right]\left[2 \alpha(1+v)-3 N_{3}(2 v-1)\right]$. It follows from (20) that at $h=h_{c r}^{c} \chi=0$, meaning that the distance $\lambda$ between the localization bands is infinite in this case. In other words, the critical condition for the continuous bifurcation corresponds to the formation of a single discrete band in an infinite body. If before reaching $h_{c r}^{c}, h$ drops rapidly (instantaneously) during deformation to $h<h_{c r}^{c}, \chi$ will be positive ( $\lambda$ has a finite value) and defined by (20). Fig. 3 shows

a

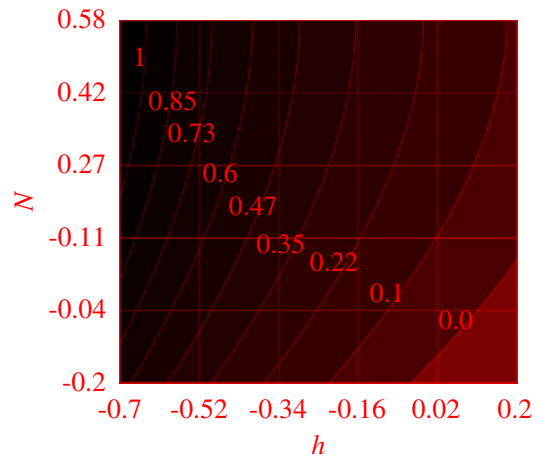

b

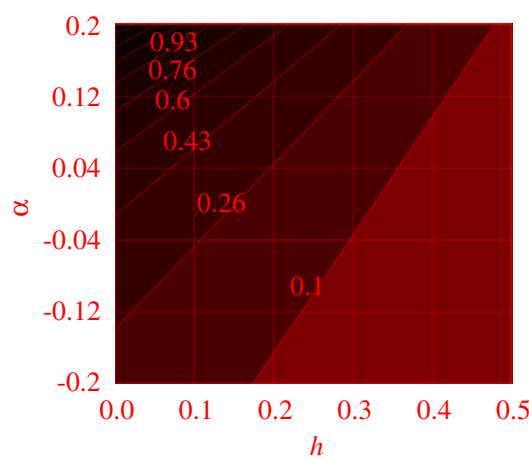

C

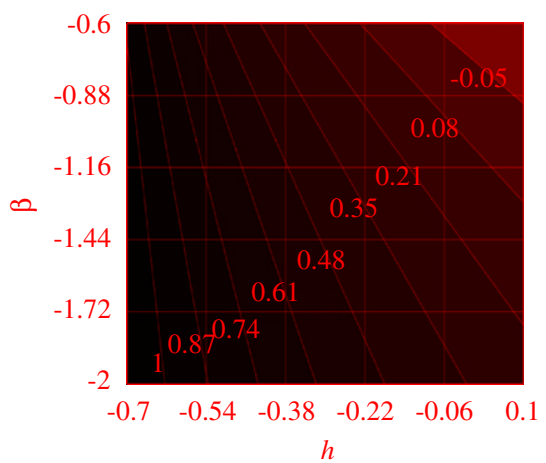

Fig. 3. Contour plots of the obtained solution $\chi(\alpha, \beta, v, N, h)$ : (a) $\chi(N, h)$ for $\alpha=-0.2$ and $\beta=-1.5$; (b) $\chi(\alpha, h)$ for $\beta=-1.5$ and $N=0.577$; (c) $\chi(\beta, h)$ for $\alpha=-0.2$ and $N=1 / \sqrt{3}$. In all cases $v=0.3$. 
a

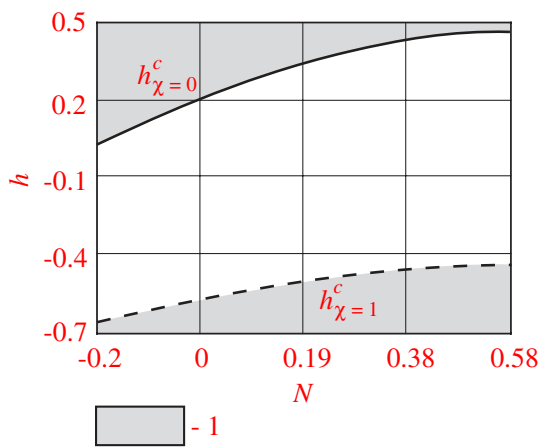

b

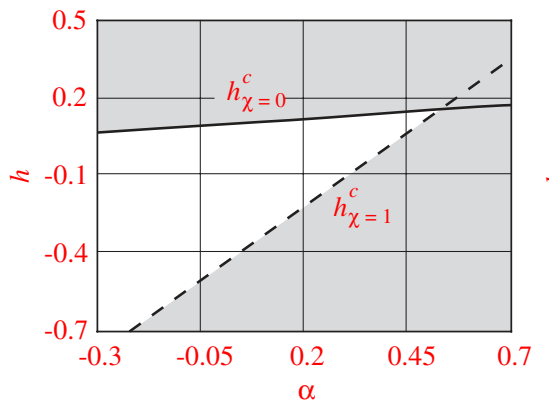

C

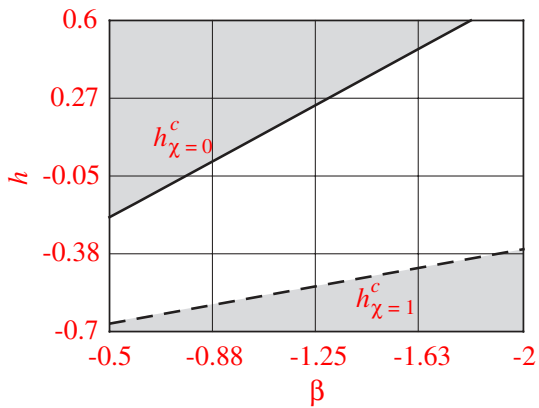

Fig. 4. Normalized hardening moduli $h_{\chi=0}^{c}=h_{\max }^{c}=h_{c r}^{c}$ and $h_{\chi=1}^{c}=h_{\min }^{c}$ versus $N$, $\alpha$ and $\beta$ : (a) $\alpha=-0.1$ and $\beta=-1.6$; (b) $\beta=-1$ and $N=0.577$; (c) $\alpha=-0.1$ and $N=1 / \sqrt{3}$. In all cases $v=0.3$. 1, Range where compaction banding is impossible.

that with the reduction of $h$, with other parameters constant, $\chi$ increases ( $\lambda$ reduces) whatever the values of these parameters. The band pattern reaches a "saturation" state at $\chi$ close to the unity, i.e., when the inter-band distance is close to the band thickness. Although it is possible that the band network forms and is "distinguishable" at $\chi>1$ ( $\lambda<d$ ), we will consider that the limit value of $\chi$ is $\chi=1$. There exists thus a limited range for the $h$ values, $h_{\chi=1}^{c} \leqslant h \leqslant h_{\chi=0}^{c}$, for which the formation of a regular band pattern is possible (Fig. 4). This range varies strongly with constitutive parameters. It can vanish or even become "negative" (Fig. 4b) when $h_{\gamma=0}^{c}=h_{c r}^{c}<h_{\chi>0}^{c}$. If this condition is met, $h$ becomes a growing function of $\chi$ (as follows from (19)) and reaches maximal value $h_{\infty}^{c}>h_{c r}^{c}$ at $\chi=\infty$ which corresponds to zero width of the unloading band. Since during the loading program, the reducing $h$ will first reach $h_{\infty}^{c}$ (and not $h_{\chi=0}^{c}=h_{c r}^{c}$ ), the localization of deformation will be impossible (the numerical models like those presented below, run in these conditions, bug).

The localization can only occur when $h_{\chi=0}^{c} \geqslant h_{\chi>0}^{c}$. Considering (19) this condition will be satisfied if either

$$
\alpha \leqslant Q, \quad \beta \leqslant Q
$$

or

$$
\alpha \geqslant Q, \quad \beta \geqslant Q,
$$

where

$$
Q=\frac{3(2 v-1)}{2((1+v)} N_{3} .
$$

Since $Q$ is always positive and the compaction banding typically develops in the geomaterials at a negative $\beta$, only the first set of inequalities in (21) may have practical sense, but it is not very limitative since the realistic $\alpha$ and $\beta$ values basically satisfy these inequalities. The condition which does impose limits on the constitutive parameters for the compaction banding to occur can be found from the same principle applied above for the case of continuous bifurcation (conditions (17)). It follows from inequality

$$
h^{c}(\chi)>h^{s h}(\chi),
$$

where $h^{\text {sh }}(\chi)$ is the critical hardening modulus for the formation of shear-band network with spacing parameter $\chi$ from Chemenda (2007). Condition (22) is much less restrictive than $h_{c r}^{c} \geqslant h_{c r}^{s h}$ applied for the continuous bifurcation. This is illustrated in Figs. 5a and c corresponding to different sets of the constitutive parameter values. Both show that the continuous bifurcation (corresponding to $h=h_{c r}$ ) can occur only in the regime of shear banding for the chosen parameter values. However, at sufficiently small $h$, compaction banding becomes possible. Since expression $h^{\text {sh }}(\chi)$ is very cumbersome (Chemenda, 2007), the condition (22) cannot be expressed in a reasonably compact explicit form. Therefore we present it graphically for two sets of the constitutive parameter values (Figs. 5b and d). These figures show that compaction banding can occur at very small negative or even positive (Fig. 5d) $\beta$ and not only at $\beta<\beta_{c r}$ as follows from (17) for the continuous bifurcation. For parameter values assumed in Figs. 5a and b, $\beta_{c r}=-1.93$, and in Figs. $5 \mathrm{c}$ and $\mathrm{d}, \beta_{c r}=1.53$ (these $\beta_{c r}$ values correspond in Figs. $5 \mathrm{~b}$ and $\mathrm{d}$ to $\Delta h_{\chi=0}=0$ ).

\section{Numerical tests}

2-D numerical simulations were performed using finite-difference, time-matching explicit code Flac3D. The Drucker-Prager model with strain softening and $\beta$ variation with $\bar{\gamma}^{p}$ was implemented in this code in C\# using User Defined Models option. 
a

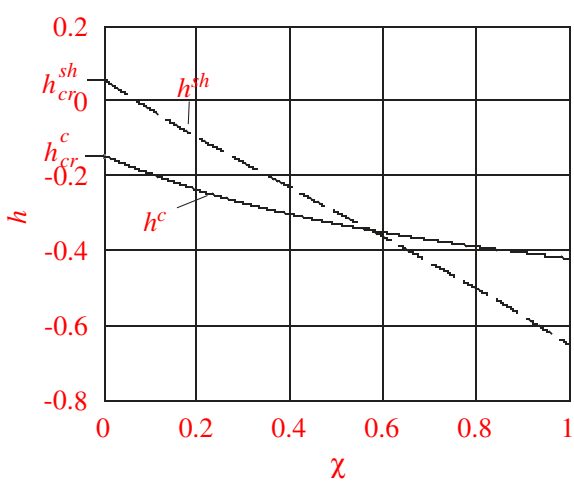

C

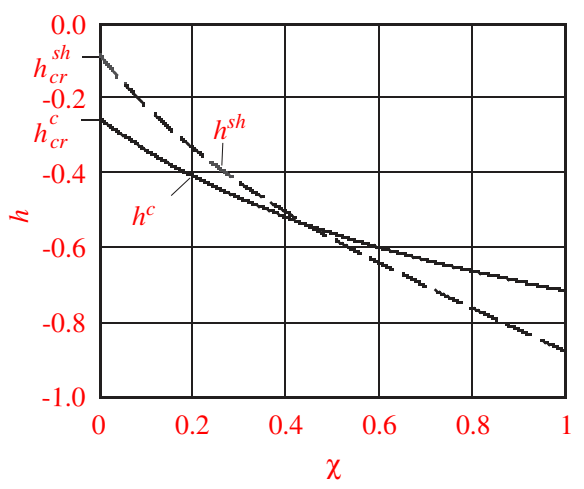

b

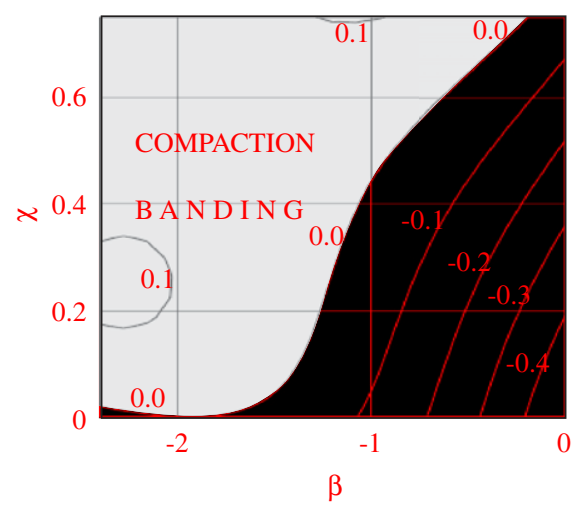

d

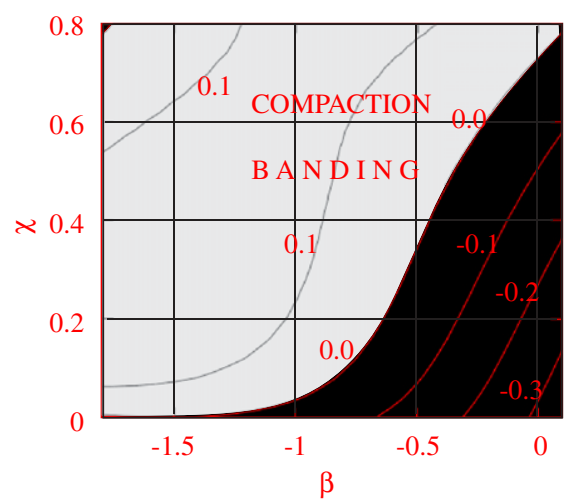

Fig. 5. (a and c) Hardening moduli for compaction $\left(h^{c}\right)$ and shear $\left(h^{\text {sh }}\right)$ banding versus spacing parameter $\%$. (b and d) Contour plots of $\Delta h(\chi, \beta)=h^{c}(\chi, \beta)-h^{s h}(\chi, \beta)$. In (a and b) $\alpha=0.2$, and $\beta=-0.7$ in (a). In (c and d) $\alpha=-0.2$ and $\beta=-0.4$ in (c). In all cases $v=0.3$ and $N=1 / \sqrt{3}$. $h^{c}(\chi, \beta)$ is from solution (19) and $h^{\text {sh }}(\chi, \beta)$, from solution in Chemenda (2007).

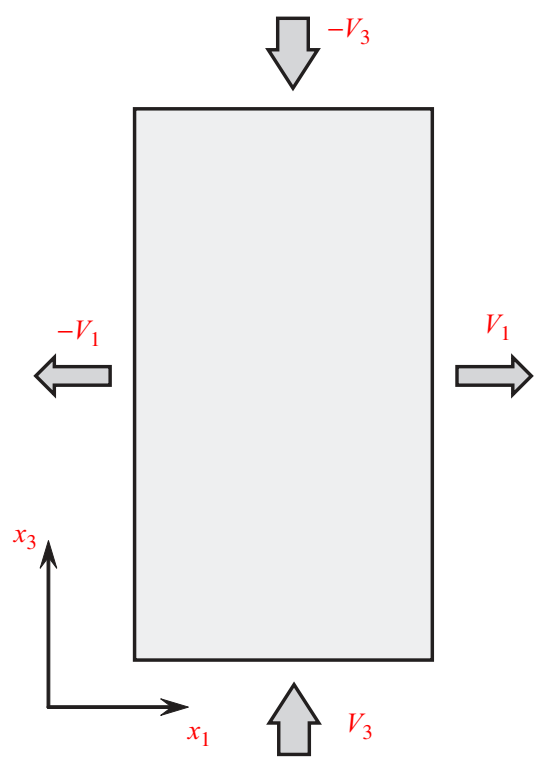

Fig. 6. Set-up of a plane-strain numerical model. The model size is $1.25 \times 5 \mathrm{~cm}(50 \times 200$ numerical zones $) ; V_{1}=0$, and $V_{3}=4 \times 10^{-14} \mathrm{~m} / \mathrm{s}$. 


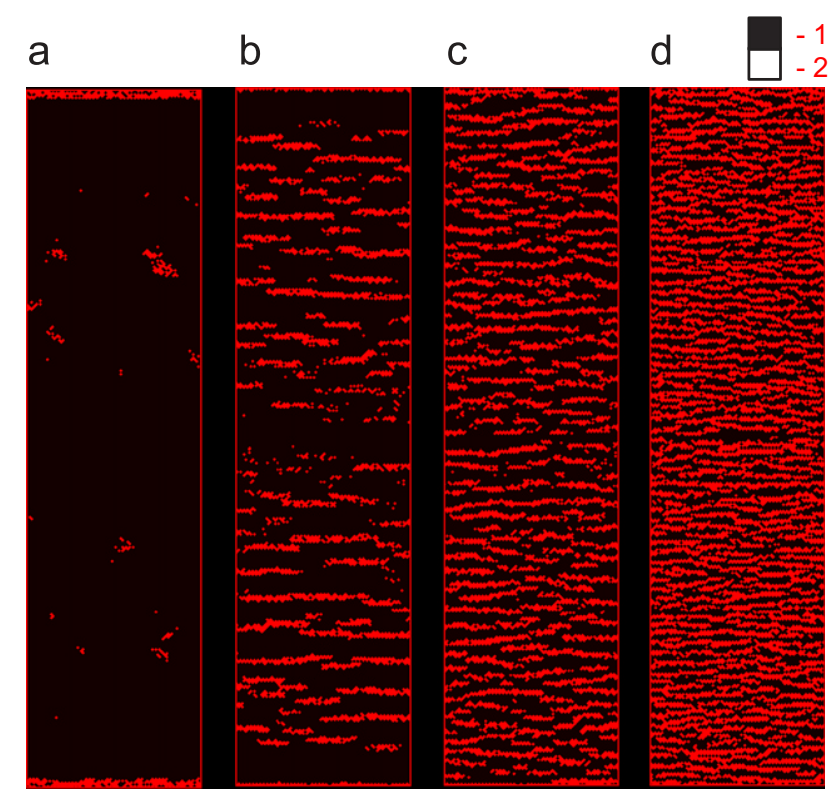

Fig. 7. Distribution of loading and unloading bands for different $h$ at $\bar{\gamma}_{\max }^{p}=3.2 \times 10^{-8}$ : (a) $h=1$; (b) $h=0.8$; (c) $h=0.6$, and (d) $h=0.4$. Other parameter values are: $\alpha=0.2, \beta=-2, N=1 / \sqrt{3}, v=0.3$. (1) Elastic-plastic state and (2) elastic state.

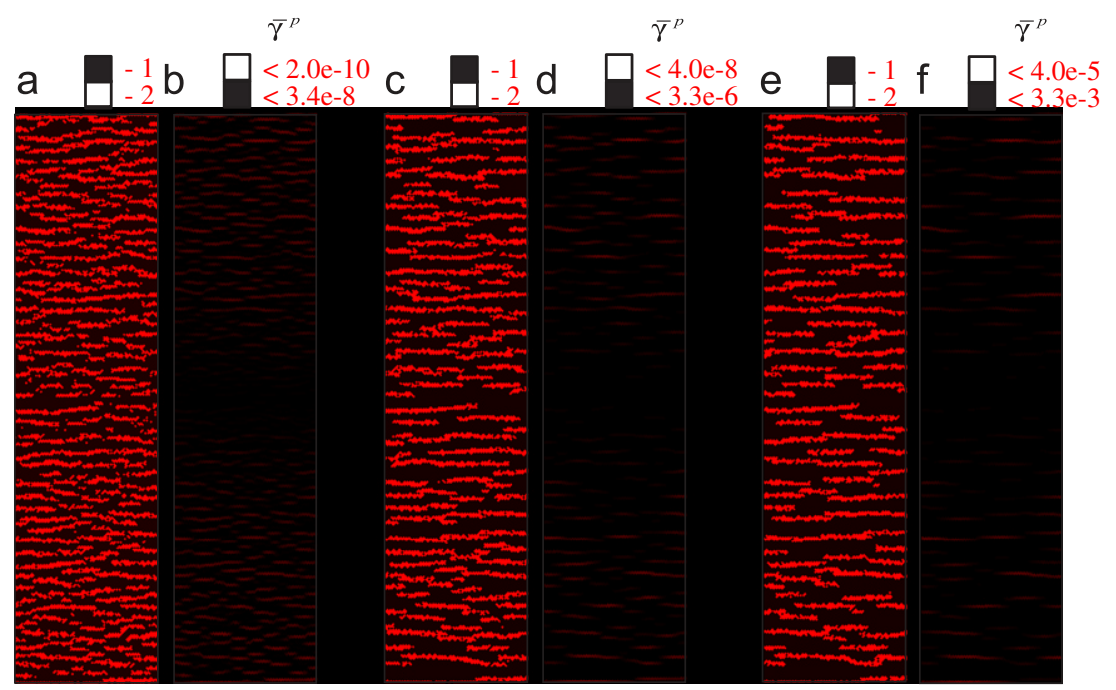

Fig. 8. Distribution of loading and unloading bands (a, c, and e), and the $\bar{\gamma}^{p}$ pattern (b, d, and f) for different $\bar{\gamma}_{\max }^{p}$ values and $h=0.6$ : (a) and (b) $\bar{\gamma}_{\max }^{p}=3.2 \times 10^{-8}$; (c) and (d) $\bar{\gamma}_{\max }^{p}=3.2 \times 10^{-6}$; (e) and (f) $\bar{\gamma}_{\max }^{p}=3.2 \times 10^{-3}$. Other parameter values are given in Fig. 7 caption. (1) Elastic-plastic state and (2) elastic state.

The numerical models are uniformly pre-stressed close to the yield surface and then subjected to slow velocity boundary conditions (in order to ensure quasi-static deformation before the bifurcation) at plane-strain state (Fig. 6). The inelastic deformation starts at the imposed (constant in the most of tests) $h$ value. There is thus a drop of $h$ from $h \gg h_{c r}^{c}$ (when no deformation bifurcation is possible) to the imposed value. In the first series of simulations only two parameters are varied in different runs, $h$ and the maximal effective inelastic shear deformation $\bar{\gamma}_{\max }^{p}$. The model is first run with $h$ close to $h_{\chi=0}^{c}=h_{c r}^{c}$. The spacing parameter $\chi$ (calculated automatically as a ratio of the total area with elastic-plastic state to the total area with elastic state) stabilizes during this run starting from $\bar{\gamma}_{\max }^{p}=3.2 \times 10^{-8}$. Other models were run for different $h$ until the same $\bar{\gamma}_{\max }^{p}$ value had been reached (Fig. 7). The influence of $\bar{\gamma}_{\max }^{p}$ on $\chi$ at different $h$ was also tested.

In the second series of simulations the impact of $h$ and $\beta$ variation with $\bar{\gamma}^{p}$ on the model deformation was studied. The last three models were conducted to test the theoretical prediction of the possibility of compaction banding at low $|\beta|$. 


\subsection{Results}

In the shear-banding simulations (Chemenda, 2007), the discontinuous bifurcation was always preceded by a very short episode of continuous bifurcation, characterized by the dense band pattern with $\chi$ approximately equal to the unity. At the next stage, the material outside the loading bands entered the elastic state. The size of the elastic (unloading) zones then rapidly grew, resulting in reduction of $\chi$ until it reached a certain stationary value at the end of this transition from continuous bifurcation to the stationary deformation pattern corresponding to the discontinuous bifurcation. The compaction banding developed in a similar way, but started directly with the discontinuous bifurcation corresponding to the transient stage with formation of a dense pattern of intermittent loading and unloading bands. The stationary deformation pattern was reached at certain (very small) $\bar{\gamma}_{\max }^{p}$ depending on $h$. Fig. 7 shows strong dependence of the distance (spacing) $\lambda$ between the bands on $h$ for the same $\bar{\gamma}_{\max }^{p}: \lambda$ reduces (the spacing parameter $\chi$ increases) with $h$ reduction. In Figs. 8a, c, and e it is seen that for $h=$ const, $\lambda$ increases with $\bar{\gamma}_{\text {max }}^{p}$, but not significantly (especially if one notes that $\bar{\gamma}_{\max }^{p}$ changes from Fig. 8a to e by five orders of magnitude) as is also seen in Fig. 9.

Fig. 10a demonstrates that starting from some resolution (density) of the numerical grid (number $n_{z}$ of the numerical zones/elements), $\chi$ can be considered constant for given $h$ and $\bar{\gamma}_{\max }^{p}$ values. The band thickness $d$, on the contrary, is directly dependent on the grid zone-size $\Delta l$ ( $\Delta l$ is the edge-length of the used cubic grid zones) and is equal to $\tilde{d} \Delta l$ where factor $\tilde{d}$ (which can be considered as the relative/normalized band thickness) as well as the whole compaction-band pattern have proved to be independent of $\Delta l$ (Fig. 10b). The $\tilde{d}$ value depends on $h$ : it is equal to $\sim 4$ at $h$ close to $h_{\chi=0}^{c}$ and reduces to $\sim 2$ with $h$ reduction to $h_{\chi=1}^{c}$.

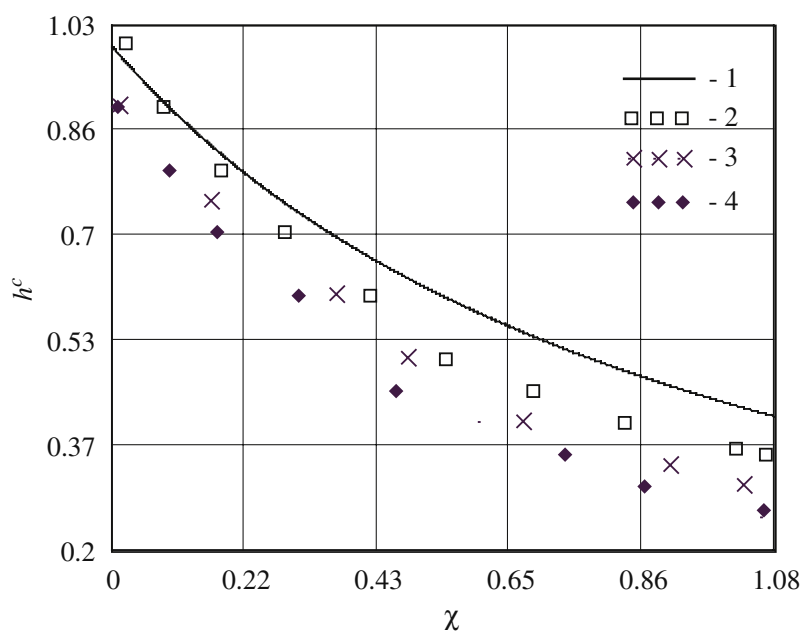

Fig. 9. Relation between the spacing parameter $\chi$ and the normalized hardening modulus $h^{c}$ : comparison of theoretical and numerical results. (1) Theoretical curve, and (2)-(4) points obtained from the numerical models for different $\bar{\gamma}_{\max }^{p}$ values: (2) $\bar{\gamma}_{\max }^{p}=3.2 \times 10^{-8} ;(3) \bar{\gamma}_{\max }^{p}=3.2 \times 10^{-6}$, and $(4)$ $\bar{\gamma}_{\max }^{p}=3.2 \times 10^{-3}$. The constitutive parameter values are given in Fig. 7 caption.

a

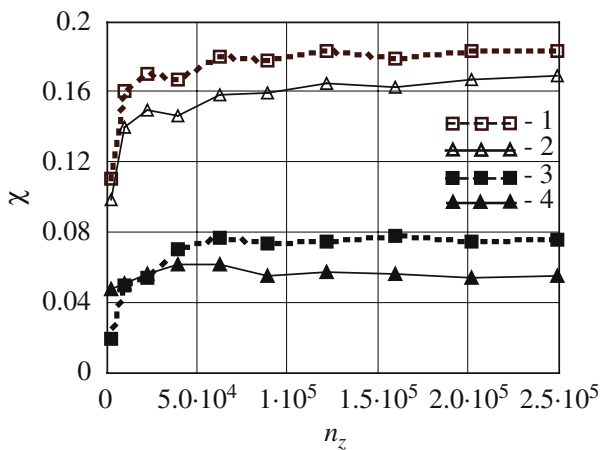

b

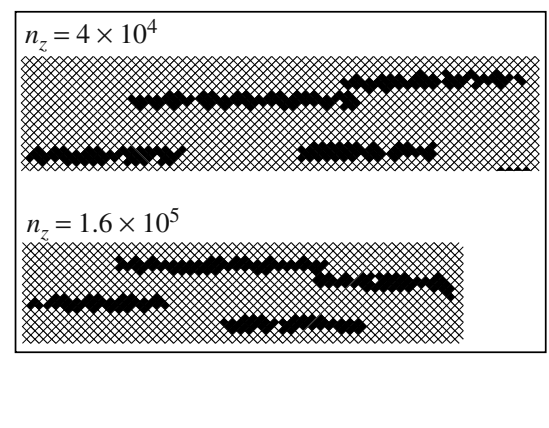

Fig. 10. (a) Variation of the spacing parameter $\chi$ with the grid density (number of the grid elements $n_{z}$ ) for different $h$ and $\bar{\gamma}_{\text {max }}^{p}$ values; (b) fragments of two numerical models (with loading bands) run under the same conditions $\left(h=0.75\right.$ and $\bar{\gamma}_{\max }^{p}=3.2 \times 10^{-6}$ ), but having different grid sizes $n_{z}$. (1) $h=0.75$ and $\bar{\gamma}_{\max }^{p}=3.2 \times 10^{-6} ;(2) h=0.75$ and $\bar{\gamma}_{\max }^{p}=3.2 \times 10^{-4} ;(3) h=0.85$ and $\bar{\gamma}_{\max }^{p}=3.2 \times 10^{-4} ;(4) h=0.85$ and $\bar{\gamma}_{\max }^{p}=3.2 \times 10^{-6}$. Other parameter values are given in Fig. 7 caption. 


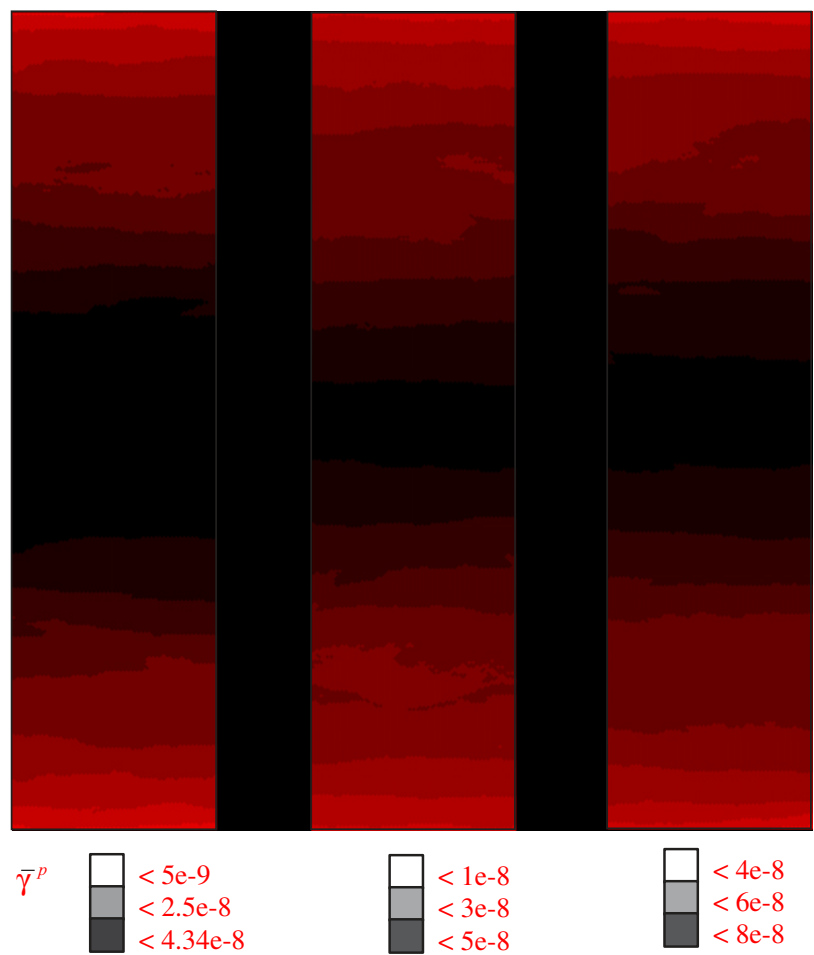

Fig. 11. Distribution of $\bar{\gamma}^{p}$ for successive stages of the model deformation in sub-critical $\left(h>h_{c r}^{c}\right)$ regime; $h=1.1\left(h_{c r}^{c}=1\right)$. Other parameter values are given in Fig. 7 caption.

If distribution of the loading and unloading bands is homogeneous through the model, the $\bar{\gamma}^{p}$ pattern shows a reduction of the deformation velocity from the model ends toward its horizontal axis (Figs. 8b, d, and f). Such a velocity gradient is also revealed in the sub-critical $\left(h>h_{c r}^{c}\right)$ regime of deformation (Fig. 11).

In real material, $h$ is not constant as in the above models and is known basically to decrease during deformation (with $\left.\bar{\gamma}^{p}\right)$. Such a reduction was introduced in the model in Fig. 12 where $h$ reduces from sub-critical value $1.1\left(h_{c r}^{c}=1\right)$ to 0.6 . At the initial stages of the model run at $h>h_{c r}^{c}$ one observes the same as in Fig. 11 gradual reduction of $\bar{\gamma}^{p}$ toward the model center (Fig. 12a) and then the rapid concentration of the deformation at the model extremities (Figs. 12b-d). This corresponds to the formation of two compaction bands similar to Fig. 7a where $h=1$ (in Fig. 12d, $h=0.6$ ). When $h$ reduces instantaneously from some sub-critical value to $h=0.6$, a dense set of bands shown in Fig. $7 \mathrm{c}$ is formed.

Like $h, \beta$ cannot remain constant during the deformation of a real material: it should normally grow (compaction cannot continue infinitely) and can become even positive at large deformation (Vajdova et al., 2004). Fig. 13 shows the result of such a growth. The deformation of this model starts with formation of two compaction bands at the model extremities (as in Fig. 7a), since initial $h$ is large (close to $h_{c r}^{c}$ and remaining constant during the model run). If $\beta$ is constant, then the banding pattern is not changed during deformation, only $\bar{\gamma}^{p}$ within the bands is increasing. Increase of $\beta$ with $\bar{\gamma}^{p}$ results in the widening of these bands (Fig. 13) which propagate toward each other and merge at a sufficiently large shortening of the model. The band widening starts when $\beta$ within the bands increases to a certain value, to -1.8 in Fig. 13 which corresponds to $\bar{\gamma}^{p} \approx 2 \times 10^{-7}$. When $\beta$ increases slower with $\bar{\gamma}^{p}$, the band widening starts correspondingly at a larger $\bar{\gamma}^{p}$.

In the models with simultaneous $h$ reduction and $\beta$ increase, $\bar{\gamma}^{p}$ variation along the model axes is smoother (in Fig. 13 the boundaries of the widening bands are sharp).

Fig. 14 shows two models run at high (compared to the continuous bifurcation prediction) dilatancy factor $\beta=-0.7$ to test the theoretical prediction presented in Fig. 5a. The models differ by the hardening moduli which are $h=-0.15$ and -0.36 and correspond (according to Fig. $5 a$ ) to shear and compaction banding, respectively. The other constitutive parameters are the same as in the previous models. The result is in total agreement with the prediction. The $\chi$ values calculated in the numerical models are almost exactly the same as those following from (20) (see graph at the top of Fig. 14).

The deformation gradient seen in Fig. 14d persists at a larger deformation if the negative $\beta$ is constant. However, it disappears ( $\bar{\gamma}^{p}$ becomes almost the same within all bands, reaching some "saturation" value of $\bar{\gamma}_{0}^{p}$ ) when $\beta$ increases with $\bar{\gamma}^{p}$. This increase results in the discrete propagation banding (Fig. 15) which is also observed in the rock samples (Fig. 16). In the model in Fig. 15, $\beta$ increases from -0.7 to 0.18 reached at $\bar{\gamma}^{p}=2 \times 10^{-7}$ at the last stage shown in Fig. 15f. This $\bar{\gamma}^{p}$ value 


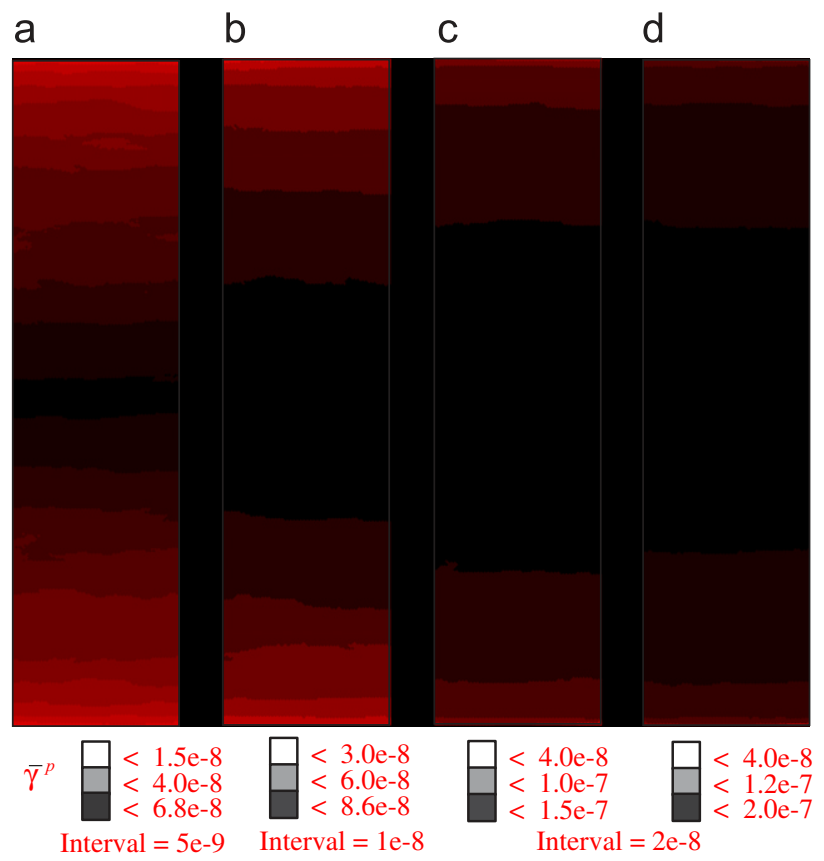

Fig. 12. $\bar{\gamma}^{p}$ pattern for successive stages of the model evolution at $\alpha=0.2, v=0.3, \beta=-2, N=1 / \sqrt{3}$, and $h$ reducing from 1.1 at $\bar{\gamma}^{p}=0-0.6$ at $\bar{\gamma}^{p}=$ $2 \times 10^{-7}$ as cosine function of $\bar{\gamma}^{p}$ : (a) $h=1.04$; (b) $h=1$; (c) $h=0.8$; (d) $h=0.6$.

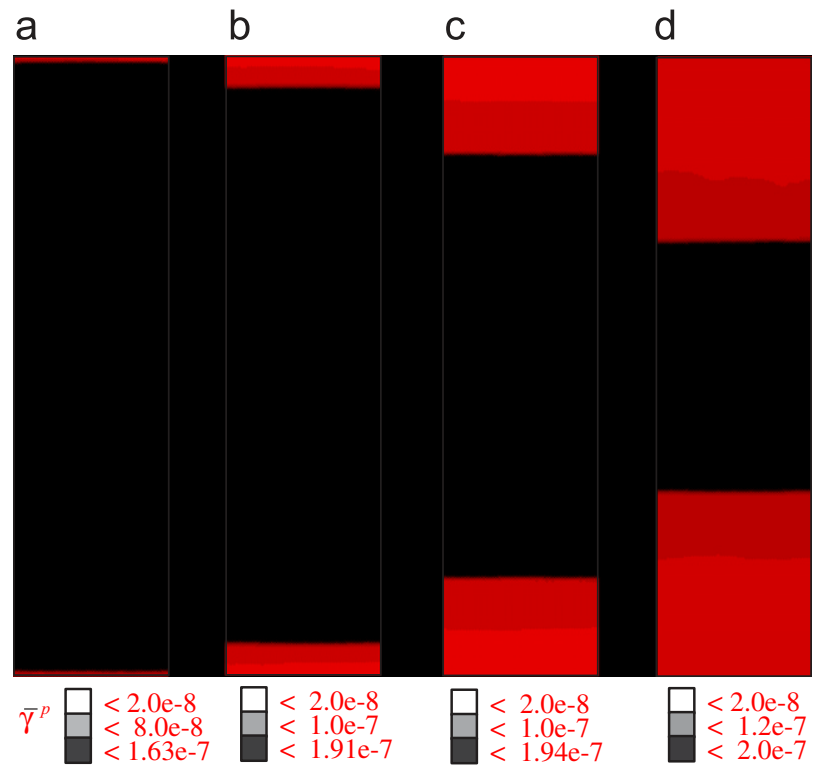

Fig. 13. $\bar{\gamma}^{p}$ pattern for successive stages of the model evolution at $\alpha=0.2, v=0.3, h=0.9, N=1 / \sqrt{3}$, and $\beta$ linearly increasing from -2 at $\bar{\gamma}^{p}=0$ to -1.8 at $\bar{\gamma}^{p}=2 \times 10^{-7}$.

corresponds to $\bar{\gamma}_{0}^{p}$ which thus is controlled by the rate of $\beta$ increase (similar to the model of continuously widening bands in Fig. 13). It is important that the compaction band set expressed in Fig. $15 \mathrm{f}$ in terms of $\bar{\gamma}^{p}$ distribution existed already (but not seen yet in terms of $\bar{\gamma}^{p}$ or damage distribution) at the very beginning of the deformation bifurcation (Fig. 15a). The "visual" effect of propagating banding occurs also when $h$ increases with $\bar{\gamma}^{p}$ at $\beta=$ const since the deformation slows down within the bands where $\bar{\gamma}^{p}$ (hence $h$ ) has reached a sufficiently large value.

Fig. 17 presents three more models having both geometry and constitutive parameter values more representative of the rock samples tested in laboratory (e.g., Baud et al., 2006; see also Fig. 16). The result is basically the same as in Fig. 14 and 


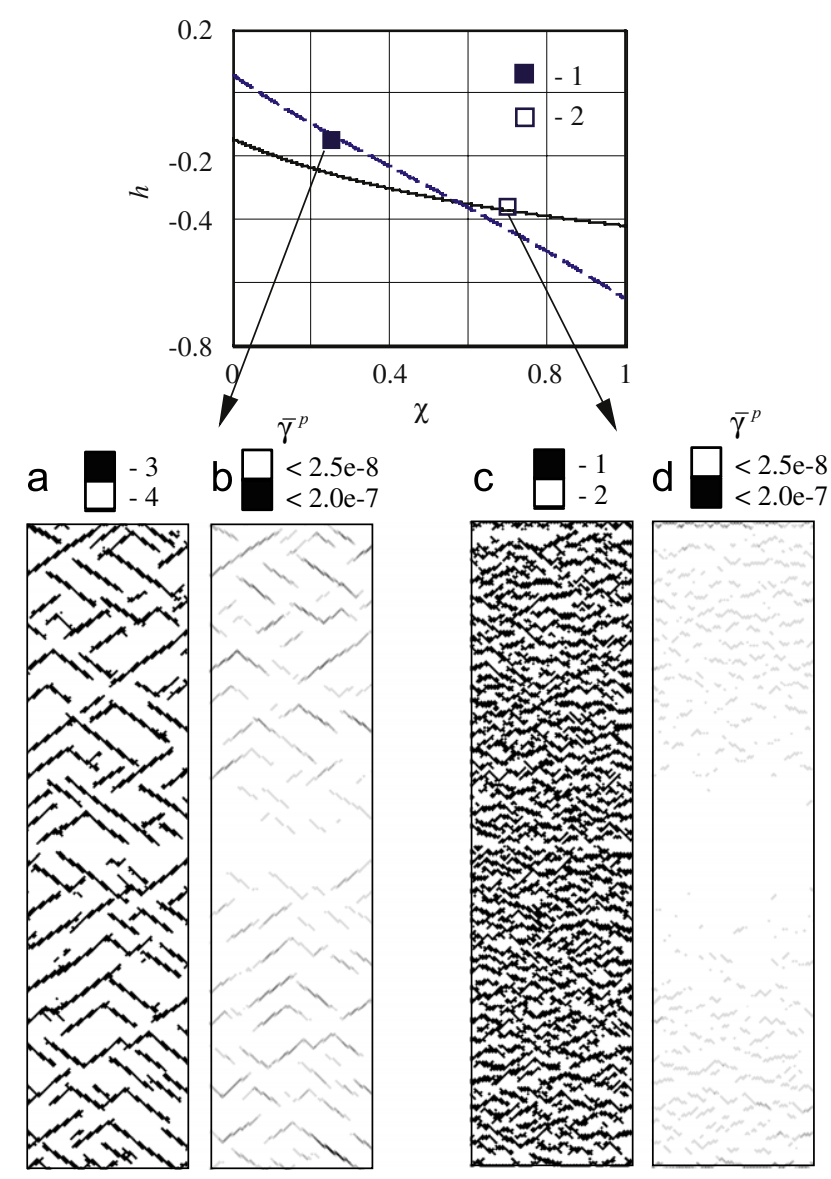

Fig. 14. Results of two numerical models run at $h=-0.15$ (a and b) and $h=-0.36$ (c and d) and the same other parameters: $\alpha=0.2, \beta=-0.7, v=0.3$, and $N=1 / \sqrt{3}$. (a) and (c) Distribution of the loading and unloading bands; (b) and (d) $\bar{\gamma}^{p}$ patterns with grey-level palettes interval of $2.5 \times 10^{-8}$. The graph at the top of this figure is a reduced copy of Fig. 5a with two points 1 and 2 added. The abscissas of these points $\chi=0.25$ and 0.71 were obtained in the above numerical models run to $\bar{\gamma}_{\max }^{p}=3.2 \times 10^{-8}$ at $h=-0.15$ and -0.36 , respectively. (1) Elastic-plastic state and (2) elastic state.

demonstrates a gradual transition from shear to compaction banding which occurs in different models with $h$ reduction $(\chi$ increase).

\section{Discussion}

\subsection{Continuous versus discontinuous bifurcation: evolution of the hardening modulus}

As in the case of shear banding (Chemenda, 2007), the formation of a set of discrete compaction bands is predicted theoretically and obtained in numerical models only in a regime of discontinuous bifurcation at $h \leqslant h_{c r}$ when the material outside the bands undergoes the elastic unloading, with inelastic deformation $\bar{\gamma}^{p}$ accelerating within the bands. This phenomenon is closely related to the evolution of the hardening modulus $h$ with $\gamma^{p}$. If $h$ reduces continuously with $\bar{\gamma}^{p}$ then the bifurcation will start in a continuous elastic-plastic regime at $h=h_{c r}$ which will be followed very rapidly by the discontinuous bifurcation as predicted by Rice and Rudnicki (1980) (the superscripts "sh" or "c" corresponding to shear and compaction banding are omitted here as this discussion is valid for all types of localization bands). As was shown above, only one band can be formed in this regime in an infinite body (the band spacing is infinite) and the material will continue to fail only within this band with further $h$ reduction. This is the case in the numerical model in Fig. 12, although instead of one band, two bands were forming at the model extremities because of its perfect homogeneity and symmetry. The same is true for shear banding. In reality, however, we observe the formation of conjugated band networks (see examples of shear bands in Chemenda, 2007) or quasi-parallel band sets (e.g., Figs. 1 and 16). According to the presented results, for such networks/sets to form, $h$ should drop rapidly (instantaneously) during deformation to $h<h_{c r}$ before reaching $h_{c r}$ as is the case in the models in Figs. 7 or 14, 15, and 17. The formation of band networks/sets both in nature and in laboratory can be considered therefore as indirect proof of a rapid $h$ drop. It remains, however, unclear whether the whole network/set is 


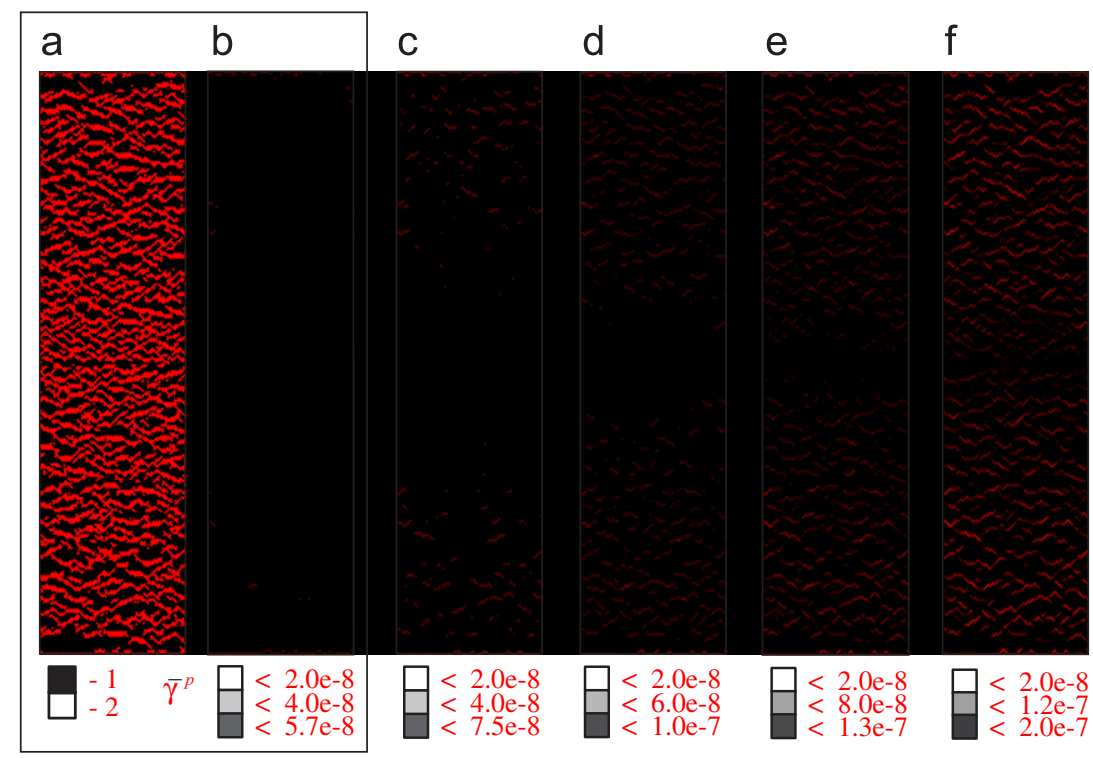

Interval $=2.0 \mathrm{e}-8$

Fig. 15. Distribution of loading and unloading bands (a) and $\bar{\gamma}^{p}$ patterns (b-f) for successive stages of the model evolution at $\alpha=0.2, v=0.3, h=-0.36$, $N=1 / \sqrt{3}$ and $\beta$ linearly increasing from -0.7 at $\bar{\gamma}^{p}=0$ to 0.18 at $\bar{\gamma}^{p}=2 \times 10^{-7}$. (a) and (b) correspond to the same stage of the model deformation. (1) Elastic-plastic state and (2) elastic state.

a

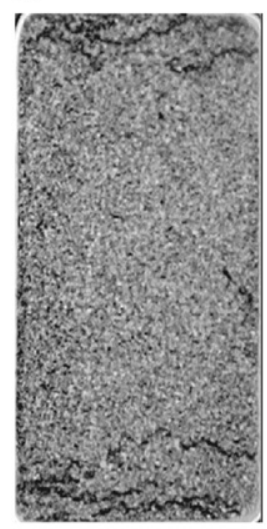

$\mathrm{b}$

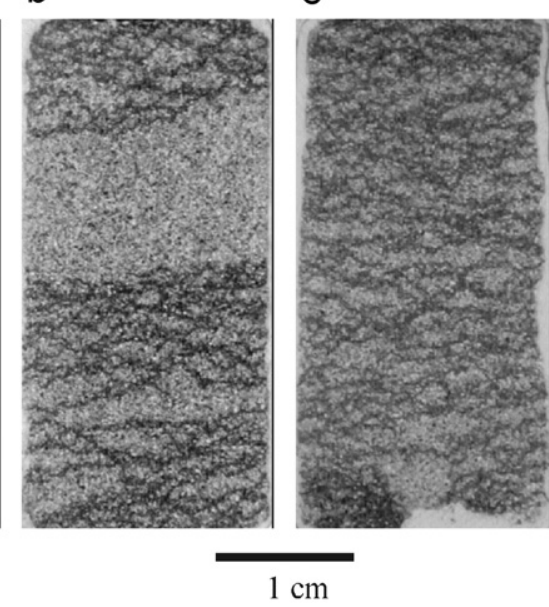

Fig. 16. Propagating discrete compaction banding in Bentheim sandstone sample under vertical axi-symmetric compression at different axial strains $\varepsilon_{v}$ (from Baud et al., 2004): (a) $\varepsilon_{v}=1.4 \times 10^{-2}$; (b) $\varepsilon_{v}=3.1 \times 10^{-2}$; (c) $\varepsilon_{v}=6 \times 10^{-2}$.

initiated instantaneously, as predicts the above theory, or not. In the laboratory tests on rock samples, in the regime of shear banding only one or two conjugated bands can usually be accommodated because of small sample size which limits $\lambda$ value. The closely spaced shear-band/fracture networks were also observed in the rock samples as mentioned above, although it is not clear whether they were generated simultaneously or not. The author observed instantaneous initiation of shear-band networks at the surface of the elastic-plastic layer under axi-symmetric extension (the layer was made of wax-type material; Fig. 9 in Chemenda et al., 2002).

The compaction band sets clearly do not appear simultaneously in the laboratory rock samples, but in a propagating manner by progressively filling the sample from the ends to the center (Klein et al., 2001; Wong et al., 2001; Baud et al., 2004) (Fig. 16). The numerical model in Fig. 15 shows, however, that it may not mean that all the bands are not initiated simultaneously, but that the bands first visually appear at the sample ends and then progressively toward its center. This propagating appearance is related to the evolution of the inelastic deformation $\bar{\gamma}^{p}$ which in reality is proportional to the material damage degree. The latter defines in turn the material texture change. Starting from some $\bar{\gamma}^{p}$ value (first attained 


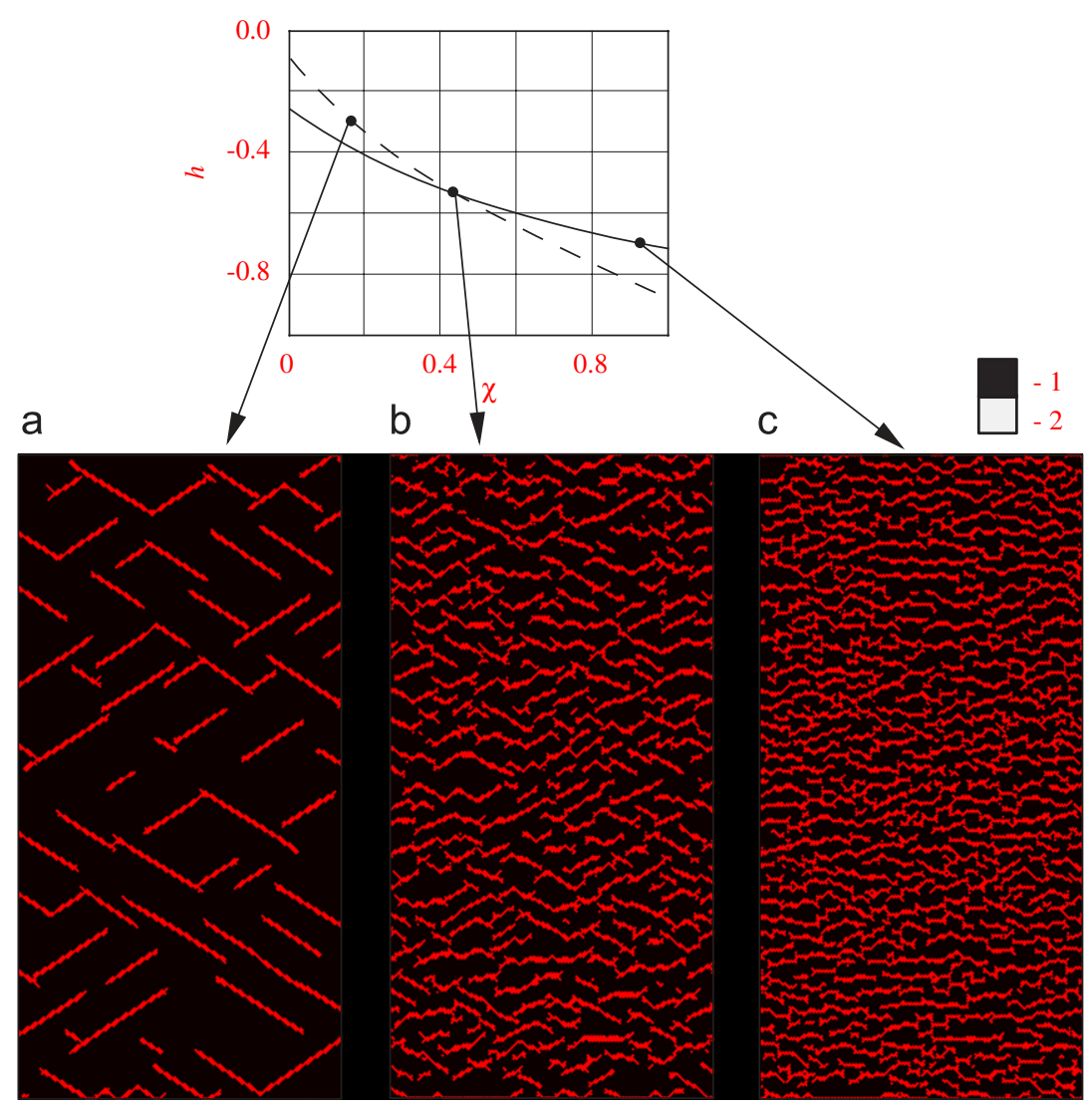

Fig. 17. Distribution of loading and unloading bands in three models deformed to $\bar{\gamma}_{\max }^{p}=2 \times 10^{-7}$ at $\alpha=-0.2, \beta=-0.4, v=0.3, E=5 \times 10^{9}$ Pa, $N=1 / \sqrt{3}$ and different $h$ values: (a) $h=-0.3$; (b) $h=-0.54$; (c) $h=-0.7$. These three cases correspond to different strain localization regimes indicated in the graph at the top of this figure which is a reduced copy of Fig. $5 c$. The model size is $2.5 \times 5 \mathrm{~cm}(100 \times 200$ numerical zones). The boundary conditions are the same as in Fig. 6. (1) Elastic-plastic state and (2) elastic state.

at the sample ends) the compaction bands become visually distinguishable which explains apparent propagating discrete banding observed in the rock samples (Fig. 16). Careful inspection of the photos in this figure reveals incipient (hardly visible) bands of damaged material in the "intact" sample segments. Yet the distribution of the of acoustic emission hypocenters during shear and compaction banding in the sandstone samples also seems to show that these processes affect the whole samples from the onset of strain localization (Fortin et al., 2006).

We thus conclude from the comparison of the laboratory experiments and field observations with theoretical analysis and numerical simulations that $h\left(\bar{\gamma}^{p}\right)$ for real materials can be discontinuous at certain $\bar{\gamma}^{p}$ value (values). This conclusion is consistent with the fact that the localization in the rock samples systematically occurs at $h>h_{c r}$ (e.g. Wong et al., 1997; Holcomb and Rudnicki, 2001) and can be characterized by the break of the stress-strain curves (see examples in Fig. 18).

What can cause an abrupt reduction of $h$ ? The function $h\left(\bar{\gamma}^{p}\right)$ is defined in the rocks by the evolution of damage within both the grains and the cement. When they are sufficiently brittle, the more or less gradual initial damage should be followed by a rapid failure of one or both components with complex interaction between them. The result will be an abrupt reduction in the dip of the stress-strain curve (like that in Fig. 18) at a certain deformation $\bar{\gamma}_{\text {lim }}^{p}$ depending on the microphysical characteristics of the material. It should be noted, however, that after the onset of the stress drop (coinciding approximately with the bifurcation onset) in the laboratory tests, the deformation of the sample is no longer homogeneous. The measured stress-strain curves record a combined response of the loading (damaging) material within the localization bands (where the stress state and material properties change very rapidly) and unloading material outside the bands. Therefore the dip of the stress-strain curve after the bifurcation point does not directly reflect the hardening modulus of the material within the band. The stress drop during abrupt $h$ reduction can be much smaller than in Fig. 18 or even be undetectable (the precision and frequency of data acquisition may be insufficient). This is a major difficulty in "extracting" the constitutive parameters after the bifurcation point. Hopefully these parameters can be accessed using numerical simulations (like those presented above) by simultaneously reproducing in the models both the sample deformation pattern and the stress-strain curves.

The parameter $\bar{\gamma}_{\text {lim }}^{p}$ is a material property and is absolutely not related to the lowest (most critical) bifurcation point (corresponding to $h_{c r}$ ) predicted from the continuous bifurcation analysis. If during loading $h_{c r}$ is not attained yet, but $\bar{\gamma}^{p}$ has 
a

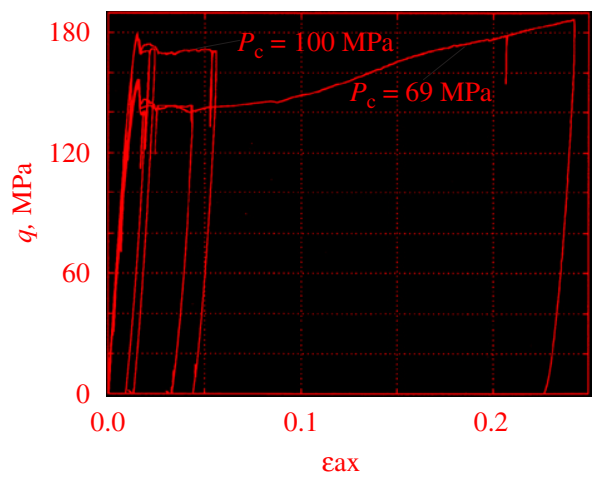

b

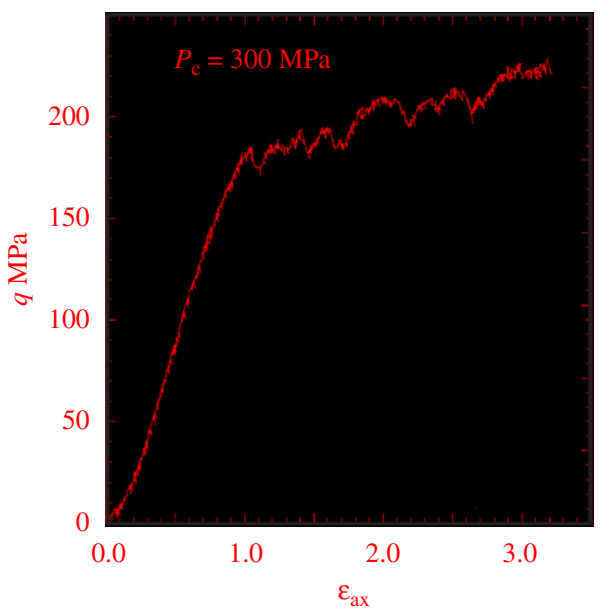

Fig. 18. Examples of experimental curves, axial differential stress $q$ versus axial strain $\varepsilon_{a x}=\left|\varepsilon_{3}\right|$, from axi-symmetric compression tests where shearcompaction and pure compaction bands were generated. $q=\left|\sigma_{3}-P_{c}\right|, P_{c}$ is the confining pressure: (a) for Bentheim sandstone (Baud et al., 2004) and (b) for Castlegate sandstone (Olsson, 1999).

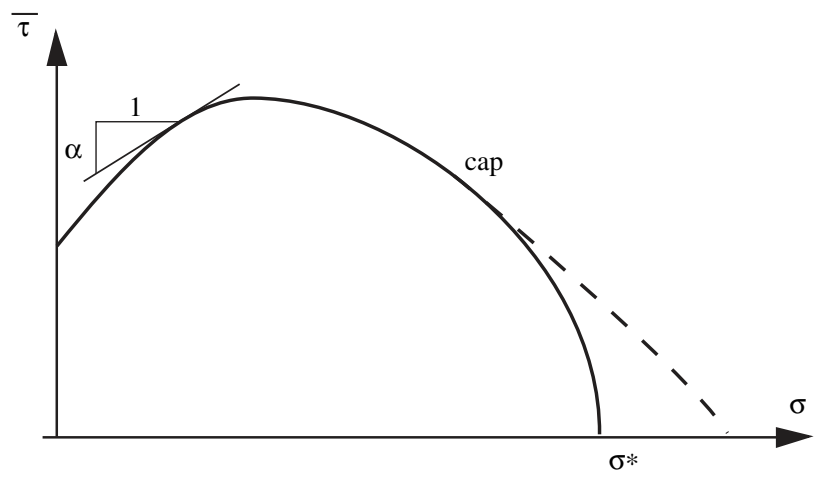

Fig. 19. Schematic of the yield surface.

already reached $\bar{\gamma}_{\text {lim }}^{p}$, the bifurcation will occur at $\bar{\gamma}^{p}=\bar{\gamma}_{\lim }^{p}$ in discontinuous mode and will result in a formation of a set of bands with a spacing being defined by $h$ value just after the point $\bar{\gamma}^{p}=\bar{\gamma}_{\text {lim. }}^{p}$. If on the contrary, $h_{c r}$ is reached before $\bar{\gamma}^{p}=\bar{\gamma}_{\text {lim }}^{p}$, then the bifurcation will start in continuous mode and be followed by discontinuous mode when $h$ (hence $\lambda$ ) is reduced sufficiently (compared to $h_{c r}$ ) for one band to be accommodated within the sample (the band can be formed only when the corresponding $\lambda$ is smaller than the sample size). Again, for one band to form, $h$ should reduce from $h_{c r}$ continuously, otherwise a set of bands will be generated. Once one band (or two bands at the sample ends) is (are) formed, the further inelastic deformation can continue only within this (these) band (bands). The abrupt drop of $h$ at this stage will not change the deformation pattern and will only accelerate the deformation within the existing bands. Increase in negative $\beta$ during such a deformation will result in widening the bands (Fig. 13) after the maximal deformation within them reached some value defined by $\beta\left(\bar{\gamma}^{p}\right)$ function (the same will result from the increase of $h\left(\bar{\gamma}^{p}\right)$ which can be negative at bifurcation and then increase with $\bar{\gamma}^{p}$ to positive value). Such a process was obtained both in the numerical model (Fig. 13) and in the rock samples (e.g. Olsson, 2001; Baud et al., 2004).

\subsection{Single-versus two-yield surface model and $\alpha$ and $\beta$ values allowing compaction banding}

The yield surface is defined by the evolution of $\bar{\tau}$ with $\sigma$ at $\bar{\gamma}^{p}=$ const when $\bar{\gamma}^{p} \neq 0$. For rock-type materials it is characterized by positive $\alpha$ at low $\sigma$, which becomes negative at the cap segment of the yield surface (Fig. 19). $\beta$ also reduces with $\sigma$ from more or less positive to negative values, but reveal strong deviation from normality and can be negative at very low $\sigma$ when $\alpha$ has its maximal positive value (e.g. Vajdova et al., 2004). It seems that deviation from normality becomes smaller at a cap when approaching $\sigma$ axis. This axis can be reached at a sharp angle (dashed line in 
Fig. 19) forming thus a vertex (see examples of the yield surfaces in Vajdova et al., 2004; Fortin et al., 2006). If a cap surface reaches hydrostatic axis at a right angle, the above constitutive formulation results in a singularity near point $\left(\bar{\tau}, \sigma^{*}\right)$ both in $\alpha$ and $\beta$ (since $\bar{\gamma}^{p}=0, \varepsilon^{p} \neq 0$ and $d \varepsilon^{p}=\beta d \bar{\gamma}^{p}$ ). This can be easily avoided by tracking the history of inelastic deformation using $\varepsilon^{p}$ instead of $\bar{\gamma}^{p}$ (Issen, 2002) as is explained below.

Issen (2002) (see also Issen and Challa, 2008) also introduced a two-yield surface model. Both surfaces correspond to Drucker-Prager yield function. The difference between the constitutive formulations consists in using two different forms of Prager consistency condition: for low $\sigma$ it is $\left(\partial f / \partial \sigma_{i j}\right) d \sigma_{i j}+\left(\partial f / \partial \bar{\gamma}^{p}\right) d \bar{\gamma}^{p}=0$ and for large $\sigma$, corresponding to a cap surface, $\left(\partial f / \partial \sigma_{i j}\right) d \sigma_{i j}+\left(\partial f / \partial \varepsilon^{p}\right) d \varepsilon^{p}=0$. The two surfaces meet at a corner (vertex) where the compaction banding is supposed to occur. Although there is no clear evidence either of a two-surface model nor of existence or absence of the vertex (Rudnicki, 2004), this model admits compaction banding at wide range of $\alpha$ and $\beta$ values (at a vertex) including those corresponding to the experimental data (the continuous bifurcation analysis for a single yield surface predicts too negative $\alpha$ and $\beta$ values (e.g., Baud et al., 2006)). On the other hand, the multi-band discontinuous bifurcation analysis in this paper predicts compaction banding at realistic $\alpha$ and $\beta$ values for a single yield surface: the maximal values of these parameters can be close to zero. In the numerical models in Fig. 17, the parameter values are typical of sandstone samples tested in laboratory: $\alpha=-0.2, \beta=-0.4, v=0.3, N=1 / \sqrt{3}$ (Baud et al., 2006). As in the rock samples, the height/width ratio in this model is of 2 . One can see the predicted gradual transition from shear to compaction banding in different tests with $h$ reduction. Not only $\beta=-0.4$ in this model is realistic (the mono-band analysis predicts $\beta_{c r} \approx-1.5$ for the same other parameters), the details of irregular zigzag shape of the bands (Figs. 17b and c and also 15) are also very similar to those observed in the samples deformed in laboratory (Fig. 16).

\subsection{Band thickness, spacing and shape}

Both theoretical analysis (Figs. 3 and 9) and numerical models (Figs. 7, 9, 14, and 17) show a rapid increase in the compaction-band spacing parameter $\chi=d / \lambda$ (reduction of spacing $\lambda$ ) with reduction of the $h$. In the numerical models $\chi$ increases somewhat more slowly and reaches the "saturation" value $\chi=1$ at an $h$ value lower than predicted (Fig. 9).

Since the constitutive formulation used involves no inherent length scale, neither $d$ nor $\lambda$ in the numerical models represents actual band thickness and spacing in a real material (even if it has exactly the same properties as the numerical model). The theory gives no indication about the absolute values of these parameters. In the numerical models they are strongly mesh dependent in the sense that they are proportional to the grid zone-size $\Delta l$. However, the ratio $\chi=d / \lambda$ in these models is practically constant (for given values of the constitutive parameters and $\bar{\gamma}_{\text {max }}^{p}$ ) and mesh-independent at sufficiently large resolution of the numerical grid (Fig. 10a). This is in accordance with the theoretical prediction. The physical origin of such mesh independence is related to the energy necessary to accelerate the inelastic deformation within the band (i.e., to form the band). This energy is supplied by the inter-band unloading (hence losing the energy) areas. The thicker the band (the larger the $\Delta l$ value) the more energy is necessary for the localization to occur; hence the larger interband area should be unloaded. Therefore both $d$ and $\lambda$ grow with $\Delta l$ increase, but their ratio remains constant (if the energy dissipating by the elastic waves in the case of dynamic deformation localisation is negligible compared to the work on an inelastic deformation).

What could not be predicted theoretically is that the relative band thickness $\tilde{d}_{m}=d_{m} / \Delta l_{m}$ in the numerical models is also mesh-independent (see, for example, Fig. 10b), i.e., it does not depend on the numerical zone-size $\Delta l_{m}$ (subscript " $m$ " stands for the model). Since both $\tilde{d}_{m}$ and $\chi_{m}$ are invariant to the mesh density, the average relative band spacing $\tilde{\lambda}_{m}=\lambda_{m} / \Delta l_{m}$ is mesh-independent as well.

The $\tilde{d}_{m}$ value in the above numerical simulations depends on $h$, but not strongly: it varies from ca. 4 to 2 when $h$ reduces from $h_{\chi=0}^{c}$ to $h_{\chi=1}^{c}$ and, on average, includes two grid zones (e.g. Fig. 10b). These $\tilde{d}_{m}$ values cannot be predicted from the used constitutive formulation and may depend on the details of the numerical solution algorithm embedded in the calculation code. $\tilde{d}_{m}$ was also shown to depend on the orientation of the grid: if the grid is parallel/orthogonal to the principal directions, the band thickness is simply equal to one zone-size. In conclusion, $\tilde{d}_{m}$ is constant in the performed simulations for given values of the constitutive parameters and does not depend on the mesh resolution (Fig. 10b) when it is sufficiently high.

In reality the thickness of an "elementary" discrete compaction band (not accumulated thickness of accreted/extending bands resulting from a large deformation) is as small as 2-3 grain sizes $\Delta l_{r}$ (e.g., Mollema and Antonellini, 1996; Baud et al., 2004), which yields for the relative band thickness $\tilde{d}_{r}=d_{r} / \Delta l_{r} 2-3$ (subscript " $r$ " corresponds to the real material). It occurs thus that $\tilde{d}_{m}$ and $\tilde{d}_{r}$ vary approximately within the same range and that roughly $\tilde{d}_{m} \approx \tilde{d}_{r}$. In other words, the band thickness in the numerical models appears to be approximately on scale with the band thickness in reality, with the scaling factor being $\Delta l_{r} / \Delta l_{m}$. The same is true for the band spacing. It follows thus that both band thickness $d_{m}$ and band spacing $\lambda_{m}$ obtained in the numerical models can be upscaled to real conditions as $d_{r} \approx\left(\Delta l_{r} / \Delta l_{m}\right) d_{m}$ and $\lambda_{r} \approx\left(\Delta l_{r} / \Delta l_{m}\right) \lambda_{m}$. It seems that the same scaling rule can be applied to the length of the band segments seen in the models (e.g., Fig. 10b) and also in nature (Fig. 1).

There is a clear difference between the geometry of compaction bands in laboratory samples (Fig. 16) and in the field (Fig. 1). In the first case the bands are thin, short, zigzag, and dense and in the second, they represent rather linear, long and thick segments. It was also noted that the material damage intensity in the "laboratory" bands is much greater than in 
natural bands (Tembe et al., 2008). All these observations are in general agreement with the obtained results. The longer, thicker and more spaced the bands in the above models are, the closer $h$ is to $h_{c r}^{c}$. In the laboratory, the spacing $\lambda$ is limited by the sample size $H$ and for the bifurcation to occur $h$ should be reduced to the value for which $\lambda<H$. Reduction of $h$ was shown in the numerical models to result in the reduction of the band thickness.

Since $h=\left(\left(d k / d \bar{\gamma}^{p}\right)-\sigma\left(d \alpha / d \bar{\gamma}^{p}\right)\right) / G$ (e.g., Chemenda, 2007), $h$ represents the rate of change of the material cohesive and/ or frictional strength with $\bar{\gamma}^{p}$. This parameter can also be viewed as the rate of material damage, with the damage degree being defined by both $h$ and $\bar{\gamma}^{p}$. Therefore, the material within the band deforming at lower $h$ will be damaged more (for the same $\bar{\gamma}^{p}$ ). The rate $\beta$ of the material compaction reduces with $\bar{\gamma}^{p}$. This parameter becomes less negative which approaches the conditions for the localization (relatively low $h$ and high $\beta$ ) to those in the models in Figs. 15 and $17 \mathrm{~b}$ and c. Since $h$ reduction can be discontinuous, the bifurcation can occur at any $\lambda<H$ including $\lambda \ll H$ as is the case in Fig. 16. To reproduce more closely the compaction-band patterns observed in the field and in the laboratory, a detailed constitutive description of the material (given rock) is necessary.

The presented numerical models were subjected to the axi-symmetric loading which corresponds to the conventional rock mechanics tests. The compaction banding was generated in the models at other stress states (other $N$ values) as well in accordance with the above theory.

\section{Conclusion}

The presented analysis of the formation of a compaction band set is very similar to shear-band network analysis (Chemenda, 2007). The case considered in this paper, however, is simpler and the obtained solutions are more exact since it was not necessary to make the usual assumption that the stresses acting in the system are small compared to the elastic-plastic moduli and that the rotational effects are negligible. As for the shear banding, the compaction-band spacing $\lambda$ is very sensitive to the normalized plastic hardening modulus $h=H / G$ and reduces from infinity to the band thickness or less when $h$ reduces from the maximal $h_{\max }$ to minimal $h_{\min }$ value. The $h$ reduction from $h \geqslant h_{\max }$ to $h_{\min }<h<h_{\max }$ during loading is rapid (instantaneous). Both $h_{\max }$ and $h_{\min }$ are defined by the constitutive parameters and the Lode-type stressstate parameter $N$. If $h$ is always larger than $h_{\max }$, the bifurcation does not occur at all. If $h$ reduces abruptly below $h_{\min }$, the deformation "jumps" into the post-localization state and the material becomes completely crushed. There exists thus a limited range for the $h$ values, $h_{\min } \leqslant h \leqslant h_{\max }$, for which the formation of a regular incipient band pattern is possible. During this process the material inside the bands undergoes accelerating inelastic deformation, while inter-band areas experience elastic unloading.

A set of compaction bands is predicted to form at realistic dilatancy factor $\beta$ values which are less negative (larger) than the values following from the continuous bifurcation analysis. Very dense compaction banding with $\lambda$ comparable to $d$ can even be formed at positive $\beta$ (Fig. 5d). The finite-difference numerical simulations confirm this result as well as the predicted rapid reduction of the band spacing $\lambda$ with $h$ (Fig. 9). Spacing $\lambda$ also varies with other constitutive and stress-state parameters: at $h=$ const $\lambda$ is minimal at $N=1 / \sqrt{3}$ (axi-symmetric compression) and increases with $N$ reduction. Yet, $\lambda$ reduces with $\alpha$ growth and $\beta$ reduction at $h=$ const (Fig. 3 ). The band shape also depends on the constitutive parameters. It is very different for different $\beta$ in numerical models (compare Fig. 10b with Figs. 15 and 17b and c). A very similar difference is observed in real materials as well (compare Figs. 1 and 16).

Although the constitutive formulation used does not contain any inherent spatial scale (either absolute or relative), it was shown that the deformation bifurcation phenomenon predicted within the frame of this formulation has a relative scale $\chi=d / \lambda$. Parameter $\chi$ was shown to be practically independent in numerical models on the grid resolution, and the ratio $d / \Delta l$ to be invariant to the grid zone-size $\Delta l$. The conclusion that follows is that both the band thickness $d_{m}$ and spacing $\lambda_{m}$ obtained in a numerical model can be upscaled to real conditions (materials) according to the following simple formulas: $d_{r} \approx\left(\Delta l_{r} / \Delta l_{m}\right) d_{m}$ and $\lambda_{r} \approx\left(\Delta l_{r} / \Delta l_{m}\right) \lambda_{m}$, where $\Delta l_{r}$ is the grain size in the real material. The same rule seems to apply to the length of the band segments. This result is important for applications of the numerical models to the natural/real material/structures.

\section{Acknowledgment}

The author is grateful to two anonymous reviewers for the useful comments.

\section{References}

Bastawros, A.-F., Bart-Smith, H., Evans, A.G., 2000. Experimental analysis of deformation mechanisms in a closed-cell aluminum alloy foam. J. Mech. Phys. Solids 48, 301-322.

Baud, P., Klein, E., Wong, T.-f., 2004. Compaction localization in porous sandstones: spatial evolution of damage and acoustic emission activity. J. Struct. Geol. 26, 603-624.

Baud, P., Vajdova, V., Wong, T.-f., 2006. Shear-enhanced compaction and strain localization: inelastic deformation and constitutive modeling of four porous sandstones. J. Geophys. Res. 111, B12401.

Bésuelle, P., 2001. Compacting and dilating shear bands in porous rocks: theoretical and experimental conditions. J. Geophys. Res. 106, 13435-13442. 
Bésuelle, P., Rudnicki, J.W., 2004. Localization: shear bands and compaction bands. In: Guéguen, Y., Boutéca, M. (Eds.), Mechanics of Fluid-Saturated Rocks. Academic Press, pp. 219-321.

Chemenda, A., Deverchere, J., Calai, E., 2002. Three-dimensional laboratory modelling of rifting: application to the Baikal Rift, Russia. Tectonophysics 356 , 253-273.

Chemenda, A., 2007. The formation of shear-band/fracture networks from a constitutive instability: theory and numerical experiment. J. Geophys. Res. 112, B11404.

Fortin, J., Stanchits, S., Dresen, G., Guéguen, Y., 2006. Acoustic emission and velocities associated with the formation of compaction bands in sandstone. J. Geophys. Res. 111, B10203.

Garagash, I.A., 1981. On the internal instability of materials with damage plasticity. Proc. Kazach. Acad. Sci. 3, 2-7 (in Russian).

Hill, R., 1962. Acceleration waves in solids. J. Mech. Phys. Solids 10, 1-16.

Holcomb, D.J., Rudnicki, J.W., 2001. Inelastic constitutive properties and shear localization in Tennessee marble. Int. J. Numer. Anal. Meth. Geomech. 25, 109-129.

Issen, K.A., 2002. The influence of constitutive models on localization conditions for porous rock. Eng. Fract. Mech. 69, $1891-1906$.

Issen, K.A., Rudnicki, J.W., 2000. Conditions for compaction bands in porous rocks. J. Geophys. Res. 105, 21,529-21,536.

Issen, K.A., Challa, V., 2008. Influence of the intermediate principal stress on the strain localization mode in porous sandstone. J. Geophys. Res. 113, B02103.

Klein, E., Baud, P., Reuschle, T., Wong, T.-f., 2001. Mechanical behaviour and failure mode of Bentheim sandstone under triaxial compression. Phys. Chem. Earth (A) 26, 21-25

Mandel, J., 1964. Propagation des surfaces de discontinuité dans un milieu élastoplastique. In: Stress Waves in Anelastic Solids. Springer-Verlag, Berlin, pp. 331-341.

Mandel, J., 1966. Conditions de stabilité et postulat de Drucker. In: Kratchenko, J., Sirieys, P.M. (Eds.), Rheology and Soil Mechanics. Springer, New York, pp. 58-68.

Mollema, P.N., Antonellini, M.A., 1996. Compaction bands: a structural analogue for anti-mode I cracks in aeolian sandstone. Tectonophysics 267, 209-228.

Nikolaevskiy, V.N., 1967. On the relation between shear and volumetric plastic deformation and shock waves in soft soils. Proc. USSR Acad. Sci. 177 (3), 542-545 (in Russian).

Olsson, W.A., 1999. Theoretical and experimental investigation of compaction bands in porous rock. J. Geophys. Res. 104, 7219-7228.

Olsson, W.A., Holcomb, D.J., 2000. Compaction localization in porous rock. Geophys. Res. Lett. 27, 3537-3540.

Olsson, W.A., 2001. Quasistatic propagation of compaction fronts in porous rock. Mech. Mater. 33, 659-668.

Ottosen, N.S., Runesson, K., 1991. Properties of discontinuous bifurcation solutions in elasto-plasticity. Int. J. Solids Struct. 27, 401-421.

Papka, S.D., Kyriakides, S., 1999. Biaxial crushing of honeycombs_Part I: experiments. Int. J. Solids Struct. 36, 4367-4396.

Perrin, G., Leblond, J.B., 1993. Rudnicki and Rice's analysis of strain localization revisited. J. Appl. Mech. 60, 842-846.

Rice, J.R., 1976. The localization of plastic deformation. In: Koiter, W.T. (Ed.), Theoretical and Applied Mechanics, Proceedings of the 14th International Congress on Theoretical and Applied Mechanics, Delft, vol. 1. North-Holland Publishing Co., pp. 207-220.

Rice, J.R., Rudnicki, J.W., 1980. A note on some features of the theory of localization of deformation. Int. J. Solids Struct. 16, 597-605.

Rudnicki, J.W., 2004. Shear and compaction band formation on an elliptic yield cap. J. Geophys. Res. 109, B03402.

Rudnicki, J.W., Rice, J.R., 1975. Conditions for the localization of deformation in pressure-sensitive dilatant materials. J. Mech. Phys. Solids 23, 371-394.

Sternlof, K., Chapin, J., Pollard, D.D., Durlofsky, L.J., 2004. Effective permeability in sandstone containing deformation band arrays. AAPG Bull. 88, 1315-1329.

Tembe, S., Baud, P., Wong, T.-f., 2008. Stress conditions for the propagation of discrete compaction bands in porous sandstone. J. Geophys. Res. 113, B09409. Vajdova, V., Baud, P., Wong, T.-f., 2004. Compaction, dilatancy, and failure in porous carbonate rocks. J. Geophys. Res. 109 , B05204.

Vardoulakis, I., Sulem, J., 1995. Bifurcation Analysis in Geomechanics. Blackie Academic and Professional, New York, 462pp.

Wong, T.-f., Baud, P., Klein, E., 2001. Localized failure modes in a compactant porous rock. Geophys. Res. Lett. 28, 2521-2524.

Wong, T.-f., David, C., Zhu, W., 1997. The transition from brittle faulting to cataclastic flow in porous sandstones: mechanical deformation. J. Geophys. Res. $102,3009-3025$ 